\title{
Angular analysis of the decay $B^{+} \rightarrow K^{+} \mu^{+} \mu^{-}$ in proton-proton collisions at $\sqrt{s}=8 \mathrm{TeV}$
}

\author{
A. M. Sirunyan et al. \\ (CMS Collaboration)
}

(Received 2 June 2018; published 20 December 2018)

\begin{abstract}
The angular distribution of the flavor-changing neutral current decay $B^{+} \rightarrow K^{+} \mu^{+} \mu^{-}$is studied in proton-proton collisions at a center-of-mass energy of $8 \mathrm{TeV}$. The analysis is based on data collected with the CMS detector at the LHC, corresponding to an integrated luminosity of $20.5 \mathrm{fb}^{-1}$. The forwardbackward asymmetry $A_{\mathrm{FB}}$ of the dimuon system and the contribution $F_{\mathrm{H}}$ from the pseudoscalar, scalar, and tensor amplitudes to the decay width are measured as a function of the dimuon mass squared. The measurements are consistent with the standard model expectations.
\end{abstract}

DOI: 10.1103/PhysRevD.98.112011

\section{INTRODUCTION}

The decay $B^{+} \rightarrow K^{+} \mu^{+} \mu^{-}$is a manifestation of a flavorchanging neutral current process of the type $b \rightarrow s \ell^{+} \ell^{-}$, with $\ell$ denoting a charged lepton. In the standard model $(\mathrm{SM})$, this decay is forbidden at tree level and occurs through higher-order processes. This makes the measurement of this process more sensitive to possible physics phenomena beyond the SM (BSM).

In the $\mathrm{SM}$, three amplitudes contribute to $B^{+} \rightarrow K^{+} \mu^{+} \mu^{-}$ via either electroweak $\mathrm{Z} / \gamma$ penguin diagrams or a $W^{+} W^{-}$ box diagram, as shown in Fig. 1. Two independent parameters describe the decay rate for the $B^{+} \rightarrow K^{+} \mu^{+} \mu^{-}$process: the forward-backward asymmetry $A_{\mathrm{FB}}$ of the dimuon system and the contribution $F_{\mathrm{H}}$ from the pseudoscalar, scalar, and tensor amplitudes to the decay width. Theoretical predictions are available for both parameters [1-3]. In the $\mathrm{SM}, A_{\mathrm{FB}}$ is zero up to small corrections, and $F_{\mathrm{H}}$ is also small. Because SM amplitudes may interfere with the contributions from BSM particles in loop diagrams, the decay can probe the presence of yet-unobserved particles and processes [4-9]. For example, a nonzero $A_{\mathrm{FB}}$ or large $F_{\mathrm{H}}$ would point to a BSM contribution $[1,10]$, which can be probed $[11,12]$ by comparing the experimental measurements with the theoretical predictions $[6,10,13]$.

In this paper, we report the measurement of $A_{\mathrm{FB}}$ and $F_{\mathrm{H}}$ as a function of the dimuon mass squared $\left(q^{2}\right)$ based on an angular fit of the decay $B^{+} \rightarrow K^{+} \mu^{+} \mu^{-}$in proton-proton collisions at $\sqrt{s}=8 \mathrm{TeV}$. Charge-conjugate decay modes

*Full author list given at the end of the article.

Published by the American Physical Society under the terms of the Creative Commons Attribution 4.0 International license. Further distribution of this work must maintain attribution to the author(s) and the published article's title, journal citation, and DOI. Funded by SCOAP ${ }^{3}$. are implied throughout this paper. The data, corresponding to an integrated luminosity of $20.5 \mathrm{fb}^{-1}$ [14], were collected by the CMS experiment at the LHC in 2012. The angular distribution of this decay has previously been studied by the BABAR [15], Belle [16], CDF [17], and LHCb [18,19] experiments, but no hints of BSM have been seen.

\section{THE CMS DETECTOR}

The central feature of the CMS detector is a superconducting solenoid of $6 \mathrm{~m}$ internal diameter, providing a magnetic field of $3.8 \mathrm{~T}$. Within the solenoid volume are a silicon pixel and a strip tracker, a lead tungstate crystal electromagnetic calorimeter, and a brass and scintillator hadron calorimeter, each composed of a barrel and two end-cap sections. Forward calorimeters extend the pseudorapidity coverage provided by the barrel and end-cap detectors. Muons are detected in gas-ionization chambers embedded in the steel flux-return yoke outside the solenoid. A detailed description of the CMS detector, together with a definition of the coordinate system used and the relevant kinematic variables, can be found elsewhere [20].

The events are selected online using a two-stage trigger system [21]. The first level is composed of custom hardware processors and uses information from the calorimeters and muon detectors to select events at a rate of around $100 \mathrm{kHz}$ within a time interval of less than $4 \mu \mathrm{s}$. The second level, known as the high-level trigger (HLT), consists of a farm of processors running a version of the full event reconstruction software optimized for fast processing, and reduces the event rate to around $1 \mathrm{kHz}$ before data storage.

\section{EVENT SELECTION}

The data for this analysis was recorded using a low-mass dimuon HLT with a displaced vertex. The trigger requires a 


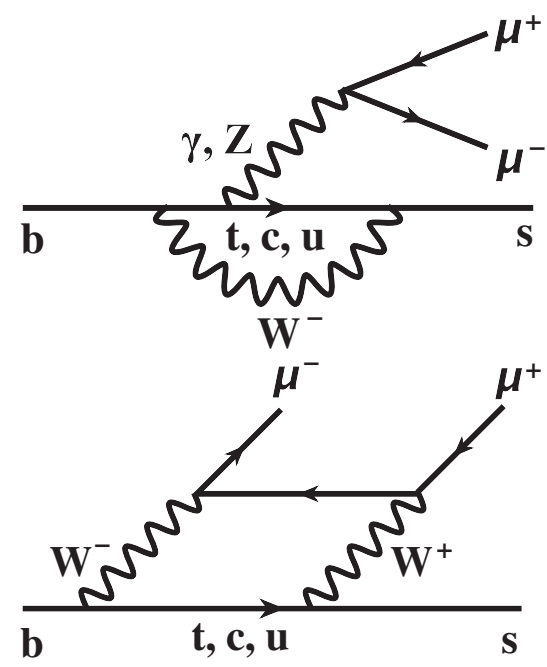

FIG. 1. The SM electroweak $\mathrm{Z} / \gamma$ penguin (left) and $W^{+} W^{-}$box (right) diagrams for the decay process $B^{+} \rightarrow K^{+} \mu^{+} \mu^{-}$.

pair of opposite-sign muons with a dimuon vertex displaced from the interaction point by more than three times the calculated uncertainty. The trigger also requires the dimuon candidate to have invariant mass in the range $1.0-4.8 \mathrm{GeV}$ and $p_{\mathrm{T}}>6.9 \mathrm{GeV}$, and for each muon to have $p_{\mathrm{T}}>3.5 \mathrm{GeV}$ and $|\eta|<2.2$.

Monte Carlo (MC) simulated event samples are widely used in the analysis. The number of simulated events for the signal sample $B^{+} \rightarrow K^{+} \mu^{+} \mu^{-}$corresponds to more than 160 times that of the data. Other simulated samples used in this analysis are $B^{+} \rightarrow K^{+} J / \psi\left(\mu^{+} \mu^{-}\right), B^{+} \rightarrow$ $K^{+} \psi(2 S)\left(\mu^{+} \mu^{-}\right)$, and $B^{+} \rightarrow \mu^{+} \mu^{-} \mathrm{X}$. In the last decay mode, the muon pairs come from $J / \psi$ or $\psi(2 S)$ decay, and $X$ denotes all other final-state particles. The MC samples are produced using the PYTHIA generator [22] version 6.424. Decays of $B^{+}$and $J / \psi$ or $\psi(2 S)$ mesons are processed by the EVTGEN [23] version 9.1 program (with the default matrix element for the signal), in which finalstate radiation is generated using PHOTOS [24]. Particles are traced through a detailed model of the detector with GEANT4 [25], producing signals similar to the actual detector responses. Particles coming from other protonproton collisions in the same or nearby beam crossings (pileup) are simulated according to the data-taking conditions, but their effects on this analysis are small.

The selected events are reconstructed through the decay into the fully charged final state of one charged hadron and a pair of oppositely charged muons. Events from the control channels $B^{+} \rightarrow K^{+} J / \psi\left(\mu^{+} \mu^{-}\right)$and $B^{+} \rightarrow K^{+} \psi(2 S)\left(\mu^{+} \mu^{-}\right)$ have the same final state as the signal process $B^{+} \rightarrow$ $K^{+} \mu^{+} \mu^{-}$, and are extensively used to validate the analysis and to evaluate the systematic uncertainties. The muons are reconstructed using information from the silicon tracker and muon detector systems [26]. They must satisfy the off-line muon identification criteria that are optimized for low- $p_{\mathrm{T}}$ muons [27]. Dimuon candidates are formed from two oppositely charged muons matching the HLT criteria that triggered the event readout. To discriminate signal events from background, additional selection criteria on kinematic variables are used. The following selection criteria are determined through a maximization of the expected signal significance using $\mathrm{MC}$ signal events and the surviving data events in the final $B^{+}$meson invariant mass fitting region, $5.1-5.6 \mathrm{GeV}$. The charged hadron track must have $p_{\mathrm{T}}>1.3 \mathrm{GeV}$ and the distance of closest approach in the transverse plane of the charged hadron trajectory to the interaction point, divided by its uncertainty, must be greater than 3.3. The $B^{+}$meson candidate is formed by combining a dimuon candidate with the charged hadron track assumed to be a kaon. The event kinematic information is updated by fitting these three tracks to a common vertex. The chi-squared probability of the vertex fit for the $B^{+}$candidate is required to be greater than $12 \%$. To further reduce the background, the distance in the transverse plane between the $B^{+}$vertex and the interaction point must be larger than 10.6 times its uncertainty. The cosine of the angle in the transverse plane between the $B^{+}$ momentum and a vector from the interaction point to the $B^{+}$meson vertex must be greater than 0.9997. After applying the selection criteria, less than $1 \%$ of the selected events contain multiple $B^{+}$candidates. In these events, only the candidate with the highest $B^{+}$decay vertex fit probability is retained.

Events with a dimuon invariant mass $(q)$ close to the $J / \psi$ or $\psi(2 S)$ resonance region are rejected to remove this contamination from the control channels, as in Ref. [28]. The $J / \psi$ and $\psi(2 S)$ resonance regions are defined as $m_{J / \psi}^{\mathrm{PDG}}-5 \sigma_{q}<q<m_{J / \psi}+3 \sigma_{q}$ and $\left|q-m_{\psi(2 S)}^{\mathrm{PDG}}\right|<5 \sigma_{q}$, respectively, where $\sigma_{q}$ is the calculated uncertainty in $q$, and the PDG superscript indicates the world-average mass value [29] for each particle. We further suppress such events by requiring, $\left|\left(m-m_{B^{+}}^{\mathrm{PDG}}\right)-\left(q-m_{J / \psi}^{\mathrm{PDG}}\right)\right|>0.13 \mathrm{GeV}$ and $\left|\left(m-m_{B^{+}}^{\mathrm{PDG}}\right)-\left(q-m_{\psi(2 S)}^{\mathrm{PDG}}\right)\right|>0.06 \mathrm{GeV}$ in the $B^{+}$ meson invariant mass region of $5.1-5.6 \mathrm{GeV}$, where $m$ is the $B^{+}$candidate invariant mass. With these requirements, the maximum contribution of events containing a $J / \psi$ or $\psi(2 S)$ is less than $7 \%$ in any $q^{2}$, and the kinematic distributions of these events can be described together with those of the combinatorial background.

\section{ANGULAR ANALYSIS}

The measurement of $A_{\mathrm{FB}}$ and $F_{\mathrm{H}}$ is performed through angular analysis in seven $q^{2}$ ranges from 1 to $22 \mathrm{GeV}^{2}$. The $q^{2}$ ranges used in this analysis are the same as in previous measurements [16-18], facilitating the comparison. The $J / \psi$ and $\psi(2 S)$ regions, corresponding to $q^{2}$ ranges of 8.68-10.09 and $12.86-14.18 \mathrm{GeV}^{2}$, respectively, are used as control regions [28,30]. Additionally, we define an inclusive low- $q^{2}$ range of $1.00-6.00 \mathrm{GeV}^{2}$ in order to 
compare the results to SM calculations with the bestcontrolled theoretical uncertainty, and a full inclusive $q^{2}$ range of $1.00-22.00 \mathrm{GeV}^{2}$, excluding the control regions. The analysis for these two ranges is performed with the same procedure as for the other ranges.

The decay rate for the process $B^{+} \rightarrow K^{+} \mu^{+} \mu^{-}$depends on $\cos \theta_{\ell}$, where $\theta_{\ell}$ is the angle between the directions of the $\mu^{-}$and $K^{+}$in the dilepton rest frame. The differential decay width $\Gamma_{\ell}$ with respected to $\cos \theta_{\ell}$ can be parametrized $[1,8,9]$ in terms of the observables of interest $A_{\mathrm{FB}}$ and $F_{\mathrm{H}}$ as:

$\frac{1}{\Gamma_{\ell}} \frac{\mathrm{d} \Gamma_{\ell}}{\mathrm{d} \cos \theta_{\ell}}=\frac{3}{4}\left(1-F_{\mathrm{H}}\right)\left(1-\cos ^{2} \theta_{\ell}\right)+\frac{1}{2} F_{\mathrm{H}}+A_{\mathrm{FB}} \cos \theta_{\ell}$

The requirement for the decay rate to remain positive over all possible lepton angles constrains the parameter space to the region $0 \leq F_{\mathrm{H}} \leq 3$ and $\left|A_{\mathrm{FB}}\right| \leq \min \left(1, F_{\mathrm{H}} / 2\right)$. The angular observables $A_{\mathrm{FB}}$ and $F_{\mathrm{H}}$ are extracted from a two-dimensional extended unbinned maximum-likelihood fit to the angular distribution of the selected $B^{+}$meson candidates in each $q^{2}$ range. The unnormalized probability density function (pdf) used in the two-dimensional fit is

$$
\begin{aligned}
\operatorname{pdf}\left(m, \cos \theta_{\ell}\right)= & Y_{\mathrm{S}} S_{\mathrm{m}}(m) S_{\mathrm{a}}\left(\cos \theta_{\ell}\right) \epsilon\left(\cos \theta_{\ell}\right) \\
& +Y_{\mathrm{B}} B_{\mathrm{m}}(m) B_{\mathrm{a}}\left(\cos \theta_{\ell}\right),
\end{aligned}
$$

where the two contributions on the right-hand side correspond to the parametrization of the signal and background. The parameters $Y_{\mathrm{S}}$ and $Y_{\mathrm{B}}$ are the yields of signal and background events, respectively. The functions $S_{\mathrm{m}}(m)$ and $S_{\mathrm{a}}\left(\cos \theta_{\ell}\right)$ describe the signal invariant mass and angular distributions, while $B_{\mathrm{m}}(m)$ and $B_{\mathrm{a}}\left(\cos \theta_{\ell}\right)$ are similar functions describing the background. The function $\epsilon\left(\cos \theta_{\ell}\right)$ is the signal efficiency as a function of $\cos \theta_{\ell}$.

The signal distribution $S_{\mathrm{m}}(m)$ is modeled as the sum of two Gaussian functions with a common mean, and $S_{\mathrm{a}}\left(\cos \theta_{\ell}\right)$ is given in Eq. (1). The background distribution $B_{\mathrm{m}}(m)$ is modeled as a single exponential function, while $B_{\mathrm{a}}\left(\cos \theta_{\ell}\right)$ is parametrized as the sum of a Gaussian function and a third- or fourth-degree polynomial, depending on the particular $q^{2}$ range.
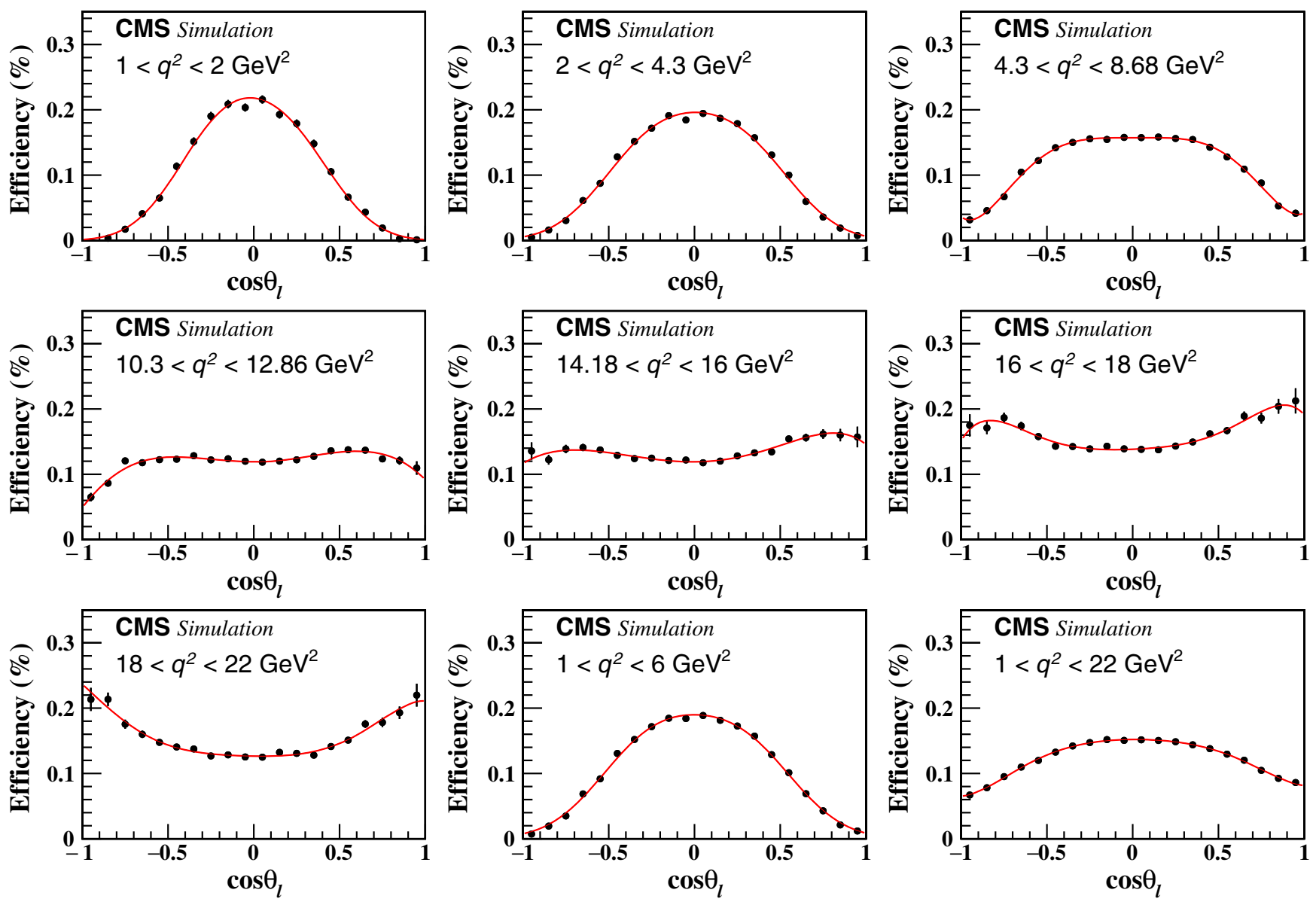

FIG. 2. The signal efficiency determined from simulated events as a function of $\cos \theta_{\ell}$ for the different $q^{2}$ ranges (points). The vertical bars indicate the statistical uncertainty. The curves show the sixth-order polynomial fits to the points. 
Many of the parameters in the final fit are set to a given value with a Gaussian constraint that reflects the input uncertainty of the value. For the $S_{\mathrm{m}}(m)$ function, the mean is constrained to the world-average $B^{+}$mass [29] and the widths and relative fraction of the two Gaussians are constrained to the values found from fitting simulated events. The parameters of the $B_{\mathrm{a}}\left(\cos \theta_{\ell}\right)$ function are obtained by fitting the events in the $B^{+}$meson invariant mass sideband regions of 5.10-5.21 and 5.35-5.46 GeV. The free parameters of the fit are $Y_{\mathrm{S}}, Y_{\mathrm{B}}, A_{\mathrm{FB}}$, and $F_{\mathrm{H}}$, as well as the exponential decay parameter of $B_{\mathrm{m}}(m)$.

The signal efficiency $\epsilon\left(\cos \theta_{\ell}\right)$ is factorized into an acceptance $\epsilon_{\text {acc }}$ times a reconstruction efficiency $\epsilon_{\text {reco }}$, which are both functions of $\cos \theta_{\ell}$. The acceptance is obtained from generated events, before the particle propagation with GEANT4, and is calculated as the fraction of MC simulated signal events passing the muon requirement of $p_{\mathrm{T}}>3.5 \mathrm{GeV}$ and $|\eta|<2.2$ relative to all generated events. It varies from 2 to $4 \%$ depending on $q^{2}$. The reconstruction efficiency is obtained from the ratio of the number of reconstructed $\mathrm{MC}$ events passing the final event selection to the number of events passing the single-muon selection at the generator level. It varies from 4 to $7 \%$ depending on $q^{2}$. The signal efficiency $\epsilon\left(\cos \theta_{\ell}\right)$ is parametrized and fit with a sixth-order polynomial, as shown in Fig. 2 for the nine different signal $q^{2}$ ranges used in this analysis.

The angular distributions of data and simulation from the two control channels are compared and the good agreement between them provides a validation of the efficiency description. We also check that the ratio of the branching fractions of the two control channels is consistent with the world-average value [29] within their uncertainties. The MC simulation samples are used to validate the fitting procedure in each $q^{2}$ range. The results of fitting the signal MC sample at the generator level and the standard signal simulation are consistent with each other. The large MC signal sample is divided into 20 subsamples and fits of
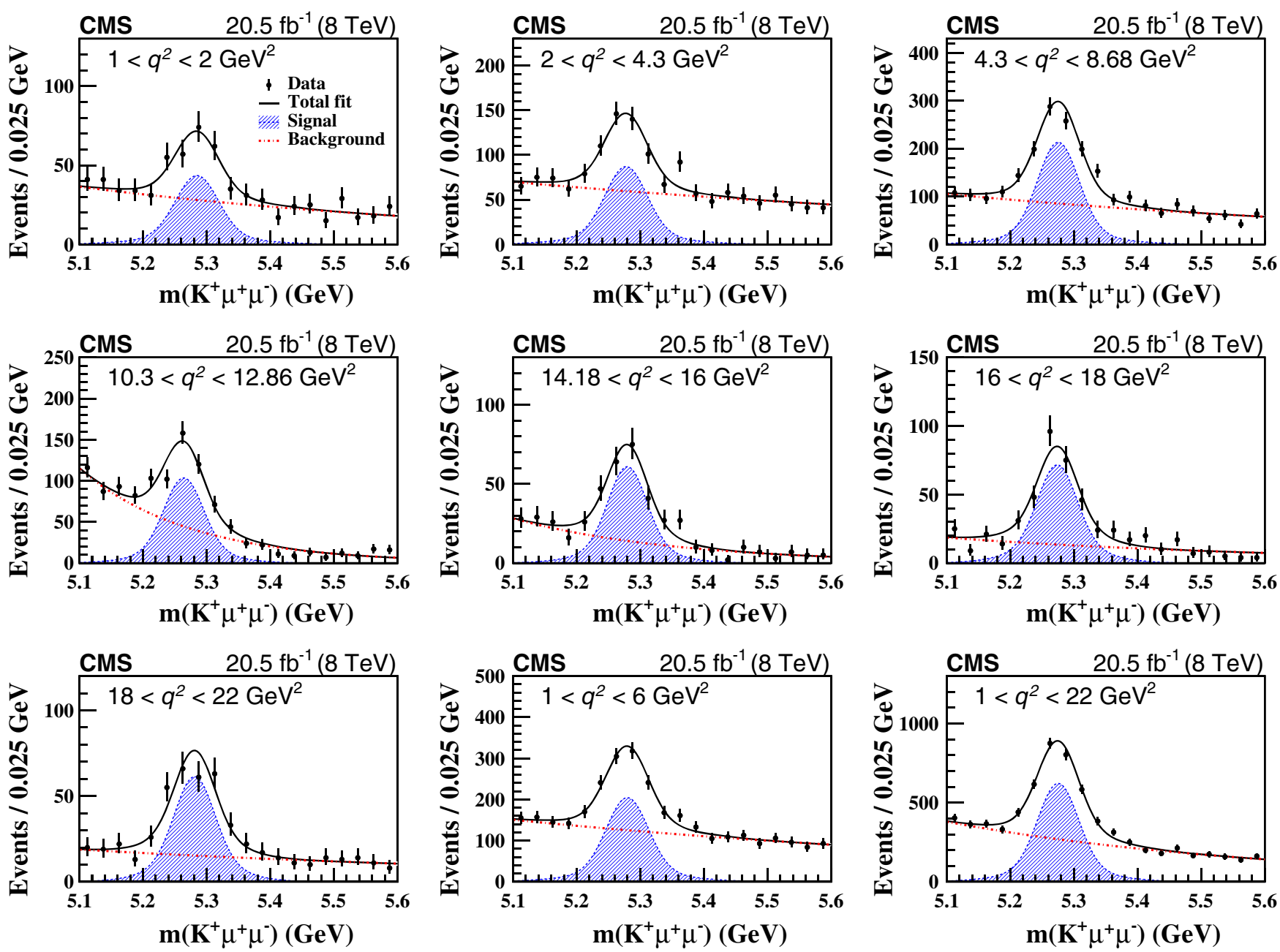

FIG. 3. Projections of the $K^{+} \mu^{+} \mu^{-}$invariant mass distributions for each $q^{2}$ range from the two-dimensional fit of data. The solid lines show the total fit, the shaded area the signal contribution, and the dashed-dotted lines the background. The vertical bars on the points represent the statistical uncertainty in the data. 

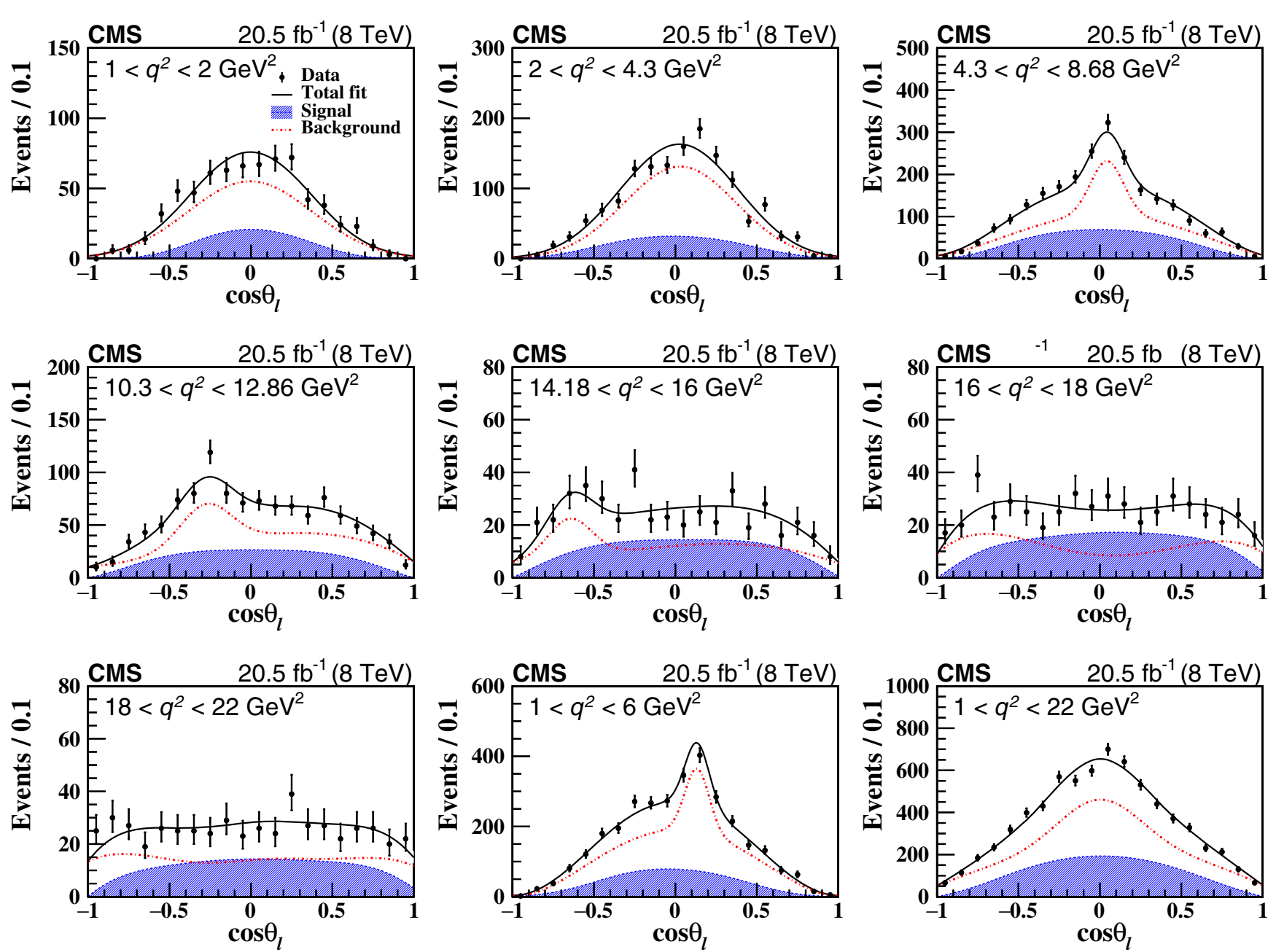

FIG. 4. Projections of the $\cos \theta_{\ell}$ distributions for each $q^{2}$ range from the two-dimensional fit of data. The solid lines show the total fit, the shaded area the signal contribution, and the dashed-dotted lines the background. The vertical bars on the points represent the statistical uncertainty in the data.

these subsamples reveal no additional bias. In addition, we generate 200 pseudoexperiments of 100 times the size of data, using the pdf in Eq. (2), with parameters from fitting the data. The differences between the fitted values from these samples and the input parameters from data follow Gaussian distributions with the means consistent with zero and the widths smaller than the variations among the signal MC subsample fits in the same $q^{2}$ range.

The final fit is performed over the full $B^{+}$meson invariant mass range and results in $2286 \pm 73$ signal events with $q^{2}$ from 1 to $22 \mathrm{GeV}^{2}$. Figures 3 and 4 show the $K^{+} \mu^{+} \mu^{-}$invariant mass and the $\cos \theta_{\ell}$ projections, respectively, for each $q^{2}$ range from the two-dimensional fit to the data.

\section{SYSTEMATIC UNCERTAINTIES}

Several sources of systematic uncertainty in the measured values of $A_{\mathrm{FB}}$ and $F_{\mathrm{H}}$ are considered, as summarized in Table I. Varying the parameter values of $S_{\mathrm{m}}(\mathrm{m})$ used to fit the signal invariant mass distribution within their uncertainties results in a negligible change in the measured values of $A_{\mathrm{FB}}$ and $F_{\mathrm{H}}$.

The finite size of the simulated event samples can affect the accuracy of the efficiency determination. To estimate the uncertainty, 200 alternative efficiency functions are created by varying the parameters of the signal efficiency function $\epsilon\left(\cos \theta_{\ell}\right)$ within their uncertainties. These alternative efficiencies are independently used to fit the data. The standard deviations of the resulting $A_{\mathrm{FB}}$ and $F_{\mathrm{H}}$ fit values are taken as their systematic uncertainties from this source. The systematic uncertainty due to the efficiency description is estimated by changing the modeling of $\epsilon\left(\cos \theta_{\ell}\right)$. The fit to $\epsilon\left(\cos \theta_{\ell}\right)$ is modified from a sixth-order polynomial to the product of a Gaussian function and a sixth-order polynomial, where the Gaussian function parameters are the fit results from $\epsilon_{\text {acc }}$, and the sixth-order polynomial parameters are the fit results from $\epsilon_{\text {reco. }}$. The differences in the results of $A_{\mathrm{FB}}$ and $F_{\mathrm{H}}$ are used as the systematic uncertainties. 
TABLE I. Absolute values of the uncertainty contributions in the measurements of $A_{\mathrm{FB}}$ and $F_{\mathrm{H}}$. For each item, the range indicates the variation of the uncertainty in the signal $q^{2}$ ranges.

\begin{tabular}{lcc}
\hline \hline Systematic uncertainty & $A_{\mathrm{FB}}\left(\times 10^{-2}\right)$ & $F_{\mathrm{H}}\left(\times 10^{-2}\right)$ \\
\hline Finite size of MC samples & $0.4-1.8$ & $0.9-5.0$ \\
Efficiency description & $0.1-1.5$ & $0.1-7.8$ \\
Simulation mismodeling & $0.1-2.8$ & $0.1-1.4$ \\
Background parametrization model & $0.1-1.0$ & $0.1-5.1$ \\
Angular resolution & $0.1-1.7$ & $0.1-3.3$ \\
Dimuon mass resolution & $0.1-1.0$ & $0.1-1.5$ \\
Fitting procedure & $0.1-3.2$ & $0.4-25$ \\
Background distribution & $0.1-7.2$ & $0.1-29$ \\
Total systematic uncertainty & $1.6-7.5$ & $4.4-39$ \\
\hline \hline
\end{tabular}

The simulated signal sample is used to evaluate the effects of any simulation mismodeling. The difference in the fitted values of $A_{\mathrm{FB}}$ and $F_{\mathrm{H}}$ between a simulated sample at the generator level without the detector simulation and reconstruction steps, and the standard signal simulation sample is assigned as the systematic uncertainty. The specific parametrization of the function used to fit the backgrounds can cause the results to change. To evaluate the effect of fitting the background $\cos \theta_{\ell}$ distribution, the degrees of the polynomials used to describe the angular shapes of the combinatorial background are decreased by one. After fitting with the alternative background parametrization, the differences in the $A_{\mathrm{FB}}$ and $F_{\mathrm{H}}$ results are taken as the systematic uncertainties from the background parametrization model. The systematic uncertainties coming from the experimental resolution in $\cos \theta_{\ell}$ and $q^{2}$ are estimated by comparing the values of $A_{\mathrm{FB}}$ and $F_{\mathrm{H}}$ obtained from the reconstructed $\mathrm{MC}$ events with those found using the generated values of $\cos \theta_{\ell}$ and $q^{2}$ in the fit.

An estimate of the systematic uncertainty from the fitting procedure is calculated using two different methods. In the first method, we divide the large simulated signal sample into multiple subsamples, each with a size similar to that of the data. The difference between the average of the fitted values of $A_{\mathrm{FB}}$ and $F_{\mathrm{H}}$ from the subsamples and the fitted value from the full sample is taken as an estimate of the systematic uncertainty from the modeling of the signal. In the second method, we generate many pseudoexperiments in which each of the mass and $\cos \theta_{\ell}$ distributions are obtained from combining a signal and background distribution. The signal distribution is obtained by selecting signal events from the simulated sample, with the number of events determined by the fit to the data. The background distribution is obtained from sampling a parent distribution that comes from subtracting the fitted signal distributions from the data. The mean value of the differences from these pseudoexperiments and the measurements from the reconstruction-level simulated signal sample is taken as an estimate of the fitting uncertainty due to the presence of background. The estimates from the two methods are then added in quadrature to obtain the overall systematic uncertainty from the fitting procedure.

In some $q^{2}$ ranges there are visible structures in the background $\cos \theta_{\ell}$ distributions, as seen in Fig. 4. We have investigated many possible contributions to these structures, and none of them has been identified. This uncertainty is estimated using the "second" method from the fitting procedure systematic uncertainty calculation, with the $\cos \theta_{\ell}$ distribution for the background obtained separately from the lower- and higher-mass sideband regions, 5.10-5.21 and 5.35-5.60 GeV. The larger of the two differences between these alternative fits and the nominal fit is taken as the systematic uncertainty from fitting the background $\cos \theta_{\ell}$ distribution.
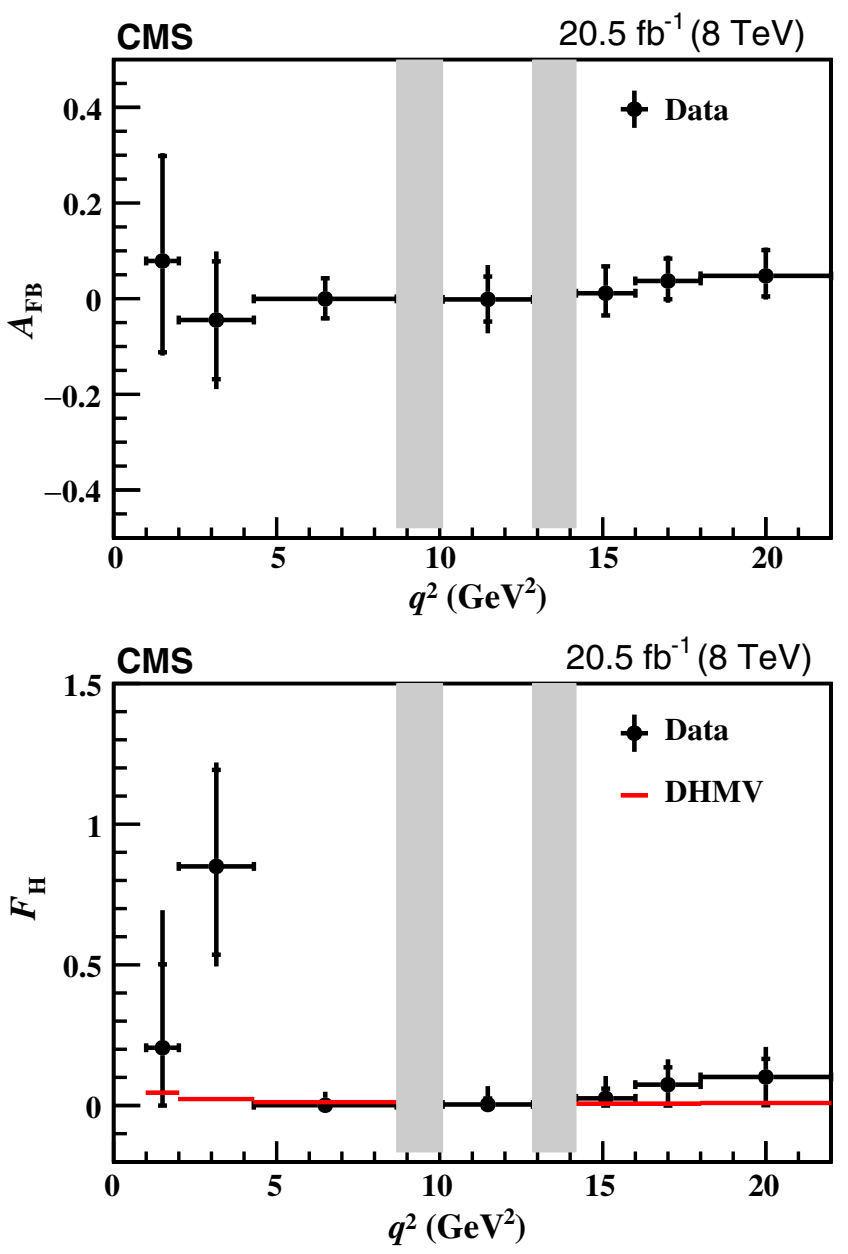

FIG. 5. Results of the $A_{\mathrm{FB}}$ (left) and $F_{\mathrm{H}}$ (right) measurements in ranges of $q^{2}$. The statistical uncertainties are shown by the inner vertical bars, while the outer vertical bars give the total uncertainties. The horizontal bars show the $q^{2}$ range widths. The vertical shaded regions are 8.68-10.09 and $12.86-14.18 \mathrm{GeV}^{2}$, corresponding to the $J / \psi$ - and $\psi(2 S)$-dominated control regions, respectively. The horizontal lines in the right plot show the DHMV SM theoretical predictions [32,33], whose uncertainties are smaller than the line width. 
TABLE II. Results of the fit for each $q^{2}$ range, together with several SM predictions. The inclusive $q^{2}=1.00-22.00 \mathrm{GeV}^{2}$ range in the bottom line does not include events from the $J / \psi$ and $\psi(2 S)$ resonance regions. The signal yield $Y_{\mathrm{S}}$ is given, along with its statistical uncertainty. The measured values of $A_{\mathrm{FB}}$ and $F_{\mathrm{H}}$ are presented, where the first uncertainties are statistical and the second are systematic. The fifth column is a theoretical prediction by C. Bobeth et al. [1,3] using the EOS package [34] with the form factors from Refs. [2,35,36]. The sixth column is the calculation from S. Descotes-Genon et al. (DHMV) based on Refs. [32,33]. The last column is the prediction using the FLAVIO package [37] with the form factors from Ref. [38]. Only the central values of the theoretical predictions are shown, since their uncertainties are insignificant compared to those in the measurements.

\begin{tabular}{|c|c|c|c|c|c|c|}
\hline$q^{2}\left(\mathrm{GeV}^{2}\right)$ & $Y_{\mathrm{S}}$ & $A_{\mathrm{FB}}$ & $F_{\mathrm{H}}$ & $F_{\mathrm{H}}(\mathrm{EOS})$ & $F_{\mathrm{H}}(\mathrm{DHMV})$ & $F_{\mathrm{H}}(\mathrm{FLAVIO})$ \\
\hline $1.00-2.00$ & $169 \pm 22$ & $0.08_{-0.19}^{+0.22} \pm 0.05$ & $0.21_{-0.21}^{+0.29} \pm 0.39$ & 0.047 & 0.046 & 0.045 \\
\hline $2.00-4.30$ & $331 \pm 32$ & $-0.04_{-0.12}^{+0.12} \pm 0.07$ & $0.85_{-0.31}^{+0.34} \pm 0.14$ & 0.024 & 0.023 & 0.022 \\
\hline $4.30-8.68$ & $785 \pm 42$ & $0.00_{-0.04}^{+0.04} \pm 0.02$ & $0.01_{-0.01}^{+0.02} \pm 0.04$ & $\cdots$ & 0.012 & 0.011 \\
\hline $10.09-12.86$ & $365 \pm 29$ & $0.00_{-0.05}^{+0.05} \pm 0.05$ & $0.01_{-0.01}^{+0.02} \pm 0.06$ & $\cdots$ & $\cdots$ & $\cdots$ \\
\hline $14.18-16.00$ & $215 \pm 19$ & $0.01_{-0.05}^{+0.06} \pm 0.02$ & $0.03_{-0.03}^{+0.03} \pm 0.07$ & 0.007 & 0.007 & 0.006 \\
\hline $16.00-18.00$ & $262 \pm 21$ & $0.04_{-0.04}^{+0.05} \pm 0.03$ & $0.07_{-0.07}^{+0.06} \pm 0.07$ & 0.007 & 0.007 & 0.006 \\
\hline $18.00-22.00$ & $226 \pm 20$ & $0.05_{-0.04}^{+0.05} \pm 0.02$ & $0.10_{-0.10}^{+0.06} \pm 0.09$ & 0.008 & 0.009 & 0.008 \\
\hline $1.00-6.00$ & $778 \pm 47$ & $-0.14_{-0.06}^{+0.07} \pm 0.03$ & $0.38_{-0.21}^{+0.17} \pm 0.09$ & 0.025 & 0.025 & 0.020 \\
\hline $1.00-22.00$ & $2286 \pm 73$ & $0.00_{-0.02}^{+0.02} \pm 0.03$ & $0.01_{-0.01}^{+0.01} \pm 0.06$ & $\ldots$ & $\cdots$ & $\ldots$ \\
\hline
\end{tabular}

The systematic uncertainties are estimated for each $q^{2}$ range independently. As the systematic uncertainty sources are considered to be independent, they are added in quadrature to obtain the total systematic uncertainties, as shown in the last row of Table I.

\section{RESULTS}

To evaluate the statistical uncertainties, the $68.3 \%$ confidence level intervals on $A_{\mathrm{FB}}$ and $F_{\mathrm{H}}$ are estimated using the profiled Feldman-Cousins technique [31]. When estimating the uncertainty in $A_{\mathrm{FB}}$ and $F_{\mathrm{H}}$, the other variable is treated as a nuisance parameter and profiled. A large number of pseudoexperiments are generated with the maximum-likelihood estimate of the nuisance parameter. The correlation between the two variables is ignored by setting the confidence interval after using this profiling method. The systematic and statistical uncertainties are added in quadrature to obtain the total uncertainty.

The measured values of $A_{\mathrm{FB}}$ and $F_{\mathrm{H}}$ for each $q^{2}$ range are shown in Fig. 5. The numerical results are summarized in Table II, including the two special $q^{2}$ ranges. The measured values of $A_{\mathrm{FB}}$ are consistent with the SM expectation of no asymmetry. Table II also includes three SM predictions for $F_{\mathrm{H}}$ with different input parameters and different handling of higher-order corrections, one of which is also shown in Fig. 5. There is generally good agreement between the predictions and our results, as well as between our results and previous measurements [15-19].

\section{SUMMARY}

An angular analysis of the decay $B^{+} \rightarrow K^{+} \mu^{+} \mu^{-}$has been performed using a data sample of proton-proton collisions corresponding to an integrated luminosity of
$20.5 \mathrm{fb}^{-1}$ recorded with the CMS detector at $\sqrt{s}=8 \mathrm{TeV}$. The forward-backward asymmetry $A_{\mathrm{FB}}$ of the muon system and the contribution $F_{\mathrm{H}}$ of the pseudoscalar, scalar, and tensor amplitudes to the decay width are measured as a function of the dimuon mass squared. The results are consistent with previous measurements, and are also compatible with three different standard model predictions.

\section{ACKNOWLEDGMENTS}

We congratulate our colleagues in the CERN accelerator departments for the excellent performance of the LHC and thank the technical and administrative staffs at CERN and at other CMS institutes for their contributions to the success of the CMS effort. In addition, we gratefully acknowledge the computing centers and personnel of the Worldwide LHC Computing Grid for delivering so effectively the computing infrastructure essential to our analyses. Finally, we acknowledge the enduring support for the construction and operation of the LHC and the CMS detector provided by the following funding agencies: BMWFW and FWF (Austria); FNRS and FWO (Belgium); CNPq, CAPES, FAPERJ, and FAPESP (Brazil); MES (Bulgaria); CERN; CAS, MoST, and NSFC (China); COLCIENCIAS (Colombia); MSES and CSF (Croatia); RPF (Cyprus); SENESCYT (Ecuador); MoER, ERC IUT, and ERDF (Estonia); Academy of Finland, MEC, and HIP (Finland); CEA and CNRS/IN2P3 (France); BMBF, DFG, and HGF (Germany); GSRT (Greece); NKFIA (Hungary); DAE and DST (India); IPM (Iran); SFI (Ireland); INFN (Italy); MSIP and NRF (Republic of Korea); LAS (Lithuania); MOE and UM (Malaysia); BUAP, CINVESTAV, CONACYT, LNS, SEP, and UASLP-FAI (Mexico); MBIE (New Zealand); PAEC (Pakistan); MSHE and NSC (Poland); FCT (Portugal); 
JINR (Dubna); MON, RosAtom, RAS and RFBR (Russia); MESTD (Serbia); SEIDI, CPAN, PCTI and FEDER (Spain); Swiss Funding Agencies (Switzerland); MST (Taipei); ThEPCenter, IPST, STAR, and NSTDA (Thailand); TUBITAK and TAEK (Turkey); NASU and SFFR (Ukraine); STFC (United Kingdom); DOE and NSF (USA). Individuals have received support from the MarieCurie program and the European Research Council and Horizon 2020 Grant, Contract No. 675440 (European Union); the Leventis Foundation; the A. P. Sloan Foundation; the Alexander von Humboldt Foundation; the Belgian Federal Science Policy Office; the Fonds pour la Formation à la Recherche dans l'Industrie et dans l'Agriculture (FRIA-Belgium); the Agentschap voor Innovatie door Wetenschap en Technologie (IWTBelgium); the F.R.S.-FNRS and FWO (Belgium) under the "Excellence of Science-EOS"-be.h Project No. 30820817; the Ministry of Education, Youth and Sports (MEYS) of the Czech Republic; the Lendület ("Momentum") Program and the János Bolyai Research Scholarship of the Hungarian Academy of Sciences, the New National Excellence Program ÚNKP, the NKFIA research Grants No. 123842, No. 123959, No. 124845, No. 124850 and No. 125105 (Hungary); the Council of Science and Industrial Research, India; the HOMING PLUS program of the Foundation for Polish Science, cofinanced from European Union, Regional Development Fund, the Mobility Plus program of the Ministry of Science and Higher Education, the National Science Center (Poland), Contracts Harmonia No. 2014/14/ M/ST2/00428, Opus No. 2014/13/B/ST2/02543, No. 2014/ 15/B/ST2/03998, and No. 2015/19/B/ST2/02861, Sonatabis No. 2012/07/E/ST2/01406; the National Priorities Research Program by Qatar National Research Fund; the Programa Estatal de Fomento de la Investigación Científica y Técnica de Excelencia María de Maeztu, Grant No. MDM-2015-0509 and the Programa Severo Ochoa del Principado de Asturias; the Thalis and Aristeia programs cofinanced by EU-ESF and the Greek NSRF; the Rachadapisek Sompot Fund for Postdoctoral Fellowship, Chulalongkorn University and the Chulalongkorn Academic into Its 2nd Century Project Advancement Project (Thailand); the Welch Foundation, Contract No. C-1845; and the Weston Havens Foundation (USA).
[1] C. Bobeth, G. Hiller, and G. Piranishvili, Angular distributions of $\mathrm{B} \rightarrow \mathrm{K}^{+} \ell^{-}$decays, J. High Energy Phys. 12 (2007) 040.

[2] A. Khodjamirian, Th. Mannel, A. A. Pivovarov, and Y. M. Wang, Charm-loop effect in $\mathrm{B} \rightarrow K^{(*)} \ell^{+} \ell^{-}$and $\mathrm{B} \rightarrow K^{*} \gamma$, J. High Energy Phys. 09 (2010) 089.

[3] C. Bobeth, G. Hiller, D. van Dyk, and C. Wacker, The decay $\mathrm{B} \rightarrow \mathrm{K} \ell^{+} \ell^{-}$at low hadronic recoil and model-independent $\delta \mathrm{B}=1$ constraints, J. High Energy Phys. 01 (2012) 107.

[4] A. Ali, T. Mannel, and T. Morozumi, Forward-backward asymmetry of dilepton angular distribution in the decay $\mathrm{b} \rightarrow \mathrm{s} \ell^{+} \ell^{-}$, Phys. Lett. B 273, 505 (1991).

[5] F. Beaujean, C. Bobeth, and S. Jahn, Constraints on tensor and scalar couplings from $\mathrm{B} \rightarrow K \bar{\mu} \mu$ and $\mathrm{B}_{\mathrm{s}} \rightarrow \bar{\mu} \mu$, Eur. Phys. J. C 75, 456 (2015).

[6] W. Altmannshofer, C. Niehoff, P. Stangl, and D. M. Straub, Status of the $\mathrm{B} \rightarrow K^{*} \mu^{+} \mu^{-}$anomaly after Moriond 2017, Eur. Phys. J. C 77, 377 (2017).

[7] W. Altmannshofer, P. Paradisi, and D. M. Straub, Modelindependent constraints on new physics in $\mathrm{b} \rightarrow \mathrm{s}$ transitions, J. High Energy Phys. 04 (2012) 008.

[8] A. Ali, P. Ball, L. T. Handoko, and G. Hiller, A comparative study of the decays $\mathrm{B} \rightarrow K^{(*)} \ell^{+} \ell^{-}$in standard model and supersymmetric theories, Phys. Rev. D 61, 074024 (2000).

[9] C. Bobeth, T. Ewerth, F. Kruger, and J. Urban, Analysis of neutral Higgs-Boson contributions to the decays $\mathrm{B}_{\mathrm{s}} \rightarrow \ell^{+} \ell^{-}$and $\mathrm{B} \rightarrow K \ell^{+} \ell^{-}$, Phys. Rev. D 64, 074014 (2001).
[10] A. K. Alok, A. Dighe, and S. Uma Sankar, Large forwardbackward asymmetry in $\mathrm{B} \rightarrow K \mu^{+} \mu^{-}$from new physics tensor operators, Phys. Rev. D 78, 114025 (2008).

[11] F. Sala and D. M. Straub, A new light particle in B decays?, Phys. Lett. B 774, 205 (2017).

[12] W. Altmannshofer and D. M. Straub, New physics in $\mathrm{b} \rightarrow \mathrm{s}$ transitions after LHC run 1, Eur. Phys. J. C 75, 382 (2015).

[13] D. Ghosh, M. Nardecchia, and S. A. Renner, Hint of lepton flavour non-universality in B meson decays, J. High Energy Phys. 12 (2014) 131.

[14] CMS Collaboration, CMS Luminosity Based on Pixel Cluster Counting-Summer 2013 Update, CMS Physics Analysis Summary Report No. CMS-PAS-LUM-13-001 (CERN, 2013).

[15] B. Aubert et al. (BABAR Collaboration), Measurements of branching fractions, rate asymmetries, and angular distributions in the rare decays $\mathrm{B} \rightarrow K \ell^{+} \ell^{-}$and $\mathrm{B} \rightarrow K^{*} \ell^{+} \ell^{-}$, Phys. Rev. D 73, 092001 (2006).

[16] J.-T. Wei et al. (Belle Collaboration), Measurement of the Differential Branching Fraction and Forward-Backward Asymmetry for $\mathrm{B} \rightarrow K^{(*)} \ell^{+} \ell^{-}$, Phys. Rev. Lett. 103, 171801 (2009).

[17] T. Aaltonen et al. (CDF Collaboration), Measurements of the Angular Distributions in the Decays B $\rightarrow K^{(*)} \mu^{+} \mu^{-}$at CDF, Phys. Rev. Lett. 108, 081807 (2012).

[18] LHCb Collaboration, Differential branching fraction and angular analysis of the $\mathrm{B}^{+} \rightarrow K^{+} \mu^{+} \mu^{-}$decay, J. High Energy Phys. 02 (2013) 105. 
[19] LHCb Collaboration, Angular analysis of charged and neutral B $\rightarrow K \mu^{+} \mu^{-}$decays, J. High Energy Phys. 05 (2014) 082 .

[20] CMS Collaboration, The CMS experiment at the CERN LHC, J. Instrum. 3, S08004 (2008).

[21] CMS Collaboration, The CMS trigger system, J. Instrum. 12, P01020 (2017).

[22] T. Sjöstrand, S. Mrenna, and P. Skands, PYTHIA 6.4 physics and manual, J. High Energy Phys. 05 (2006) 026.

[23] D. J. Lange, The EvtGen particle decay simulation package, Nucl. Instrum. Methods Sect. A 462, 152 (2001).

[24] P. Golonka and Z. Was, PHOTOS monte carlo: A precision tool for QED corrections in $Z$ and $W$ decays, Eur. Phys. J. C 45, 97 (2006).

[25] S. Agostinelli et al. (GEANT4 Collaboration), GEANT4-A simulation toolkit, Nucl. Instrum. Methods Sect. A 506, 250 (2003).

[26] CMS Collaboration, Performance of CMS muon reconstruction in pp collision events at $\sqrt{s}=7 \mathrm{TeV}$, J. Instrum. 7, P10002 (2012).

[27] CMS Collaboration, Muon ID performance: low- $p_{\mathrm{T}}$ muon efficiencies, CMS Detector Performance Report No. CMSDP-2014-020 (CERN, 2014).

[28] CMS Collaboration, Angular analysis and branching fraction measurement of the decay $\mathrm{B}^{0} \rightarrow K^{* 0} \mu^{+} \mu^{-}$, Phys. Lett. B 727, 77 (2013).

[29] C. Patrignani et al. (Particle Data Group), Review of particle physics, Chin. Phys. C 40, 100001 (2016).
[30] CMS Collaboration, Angular analysis of the decay $\mathrm{B}^{0} \rightarrow$ $K^{* 0} \mu^{+} \mu^{-}$from pp collisions at $\sqrt{s}=8 \mathrm{TeV}$, Phys. Lett. B 753, 424 (2016).

[31] G. J. Feldman and R. D. Cousins, A unified approach to the classical statistical analysis of small signals, Phys. Rev. D 57, 3873 (1998).

[32] S. Descotes-Genon, L. Hofer, J. Matias, and J. Virto, On the impact of power corrections in the prediction of $\mathrm{B} \rightarrow K^{*} \mu^{+} \mu^{-}$observables, J. High Energy Phys. 12 (2014) 125.

[33] S. Descotes-Genon, L. Hofer, J. Matias, and J. Virto, Global analysis of $\mathrm{b} \rightarrow \mathrm{s} \ell \ell$ anomalies, J. High Energy Phys. 06 (2016) 092.

[34] D. van Dyk et al., EOS-A HEP program for flavor observables (2016), https: //eos.github.io.

[35] C. Bouchard, G. P. Lepage, C. Monahan, H. Na, and J. Shigemitsu (HPQCD Collaboration), Rare decay $\mathrm{B} \rightarrow$ $K \ell^{+} \ell^{-}$form factors from lattice QCD, Phys. Rev. D 88, 054509 (2013); 88, 079901(E) (2013).

[36] P. Ball and R. Zwicky, New results on B $\rightarrow \pi, K, \eta$ decay form factors from light-cone sum rules, Phys. Rev. D 71, 014015 (2005).

[37] D. M. Straub, flavio: a Python package for flavour and precision phenomenology in the Standard Model and beyond, arXiv:1810.08132.

[38] J. A. Bailey et al. (Fermilab Lattice and MILC Collaborations), $\mathrm{B} \rightarrow K \ell^{+} \ell^{-}$decay form factors from three-flavor lattice QCD, Phys. Rev. D 93, 025026 (2016).

A. M. Sirunyan, ${ }^{1}$ A. Tumasyan, ${ }^{1}$ W. Adam, ${ }^{2}$ F. Ambrogi, ${ }^{2}$ E. Asilar, ${ }^{2}$ T. Bergauer, ${ }^{2}$ J. Brandstetter, ${ }^{2}$ E. Brondolin, ${ }^{2}$ M. Dragicevic, ${ }^{2}$ J. Erö, ${ }^{2}$ A. Escalante Del Valle, ${ }^{2}$ M. Flechl, ${ }^{2}$ R. Frühwirth, ${ }^{2, b}$ V. M. Ghete, ${ }^{2}$ J. Hrubec, ${ }^{2}$ M. Jeitler, ${ }^{2, b}$ N. Krammer, ${ }^{2}$ I. Krätschmer, ${ }^{2}$ D. Liko, ${ }^{2}$ T. Madlener, ${ }^{2}$ I. Mikulec, ${ }^{2}$ N. Rad, ${ }^{2}$ H. Rohringer, ${ }^{2}$ J. Schieck, ${ }^{2, b}$ R. Schöfbeck, ${ }^{2}$ M. Spanring, ${ }^{2}$ D. Spitzbart, ${ }^{2}$ A. Taurok, ${ }^{2}$ W. Waltenberger, ${ }^{2}$ J. Wittmann, ${ }^{2}$ C.-E. Wulz, ${ }^{2, b}$ M. Zarucki, ${ }^{2}$ V. Chekhovsky, ${ }^{3}$ V. Mossolov, ${ }^{3}$ J. Suarez Gonzalez, ${ }^{3}$ E. A. De Wolf, ${ }^{4}$ D. Di Croce, ${ }^{4}$ X. Janssen, ${ }^{4}$ J. Lauwers, ${ }^{4}$ M. Pieters, ${ }^{4}$

M. Van De Klundert, ${ }^{4}$ H. Van Haevermaet, ${ }^{4}$ P. Van Mechelen, ${ }^{4}$ N. Van Remortel, ${ }^{4}$ S. Abu Zeid, ${ }^{5}$ F. Blekman, ${ }^{5}$ J. D'Hondt, ${ }^{5}$ I. De Bruyn, ${ }^{5}$ J. De Clercq, ${ }^{5}$ K. Deroover, ${ }^{5}$ G. Flouris, ${ }^{5}$ D. Lontkovskyi, ${ }^{5}$ S. Lowette, ${ }^{5}$ I. Marchesini, ${ }^{5}$ S. Moortgat, ${ }^{5}$ L. Moreels, ${ }^{5}$ Q. Python, ${ }^{5}$ K. Skovpen, ${ }^{5}$ S. Tavernier, ${ }^{5}$ W. Van Doninck, ${ }^{5}$ P. Van Mulders,${ }^{5}$ I. Van Parijs, ${ }^{5}$ D. Beghin, ${ }^{6}$ B. Bilin, ${ }^{6}$ H. Brun, ${ }^{6}$ B. Clerbaux,${ }^{6}$ G. De Lentdecker, ${ }^{6}$ H. Delannoy, ${ }^{6}$ B. Dorney, ${ }^{6}$ G. Fasanella, ${ }^{6}$ L. Favart, ${ }^{6}$ R. Goldouzian, ${ }^{6}$ A. Grebenyuk, ${ }^{6}$ A. K. Kalsi, ${ }^{6}$ T. Lenzi,${ }^{6}$ J. Luetic, ${ }^{6}$ N. Postiau, ${ }^{6}$ E. Starling, ${ }^{6}$ L. Thomas, ${ }^{6}$ C. Vander Velde, ${ }^{6}$ P. Vanlaer, ${ }^{6}$ D. Vannerom, ${ }^{6}$ Q. Wang, ${ }^{6}$ T. Cornelis, ${ }^{7}$ D. Dobur, ${ }^{7}$ A. Fagot,${ }^{7}$ M. Gul, ${ }^{7}$ I. Khvastunov, ${ }^{7, c}$ D. Poyraz, ${ }^{7}$ C. Roskas, ${ }^{7}$ D. Trocino, ${ }^{7}$ M. Tytgat, ${ }^{7}$ W. Verbeke, ${ }^{7}$ B. Vermassen, ${ }^{7}$ M. Vit, ${ }^{7}$ N. Zaganidis, ${ }^{7}$ H. Bakhshiansohi,${ }^{8}$ O. Bondu, ${ }^{8}$ S. Brochet,${ }^{8}$ G. Bruno, ${ }^{8}$ C. Caputo, ${ }^{8}$ P. David, ${ }^{8}$ C. Delaere, ${ }^{8}$ M. Delcourt, ${ }^{8}$ B. Francois, ${ }^{8}$ A. Giammanco, ${ }^{8}$ G. Krintiras,${ }^{8}$ V. Lemaitre, ${ }^{8}$ A. Magitteri, ${ }^{8}$ A. Mertens, ${ }^{8}$ M. Musich, ${ }^{8}$ K. Piotrzkowski, ${ }^{8}$ A. Saggio, ${ }^{8}$ M. Vidal Marono, ${ }^{8}$ S. Wertz, ${ }^{8}$ J. Zobec, ${ }^{8}$ F. L. Alves, ${ }^{9}$ G. A. Alves, ${ }^{9}$ L. Brito, ${ }^{9}$ G. Correia Silva, ${ }^{9}$ C. Hensel,,${ }^{9}$ A. Moraes, ${ }^{9}$ M. E. Pol,,${ }^{9}$ P. Rebello Teles, ${ }^{9}$ E. Belchior Batista Das Chagas, ${ }^{10}$ W. Carvalho, ${ }^{10}$ J. Chinellato, ${ }^{10, \mathrm{~d}}$ E. Coelho, ${ }^{10}$ E. M. Da Costa, ${ }^{10}$ G. G. Da Silveira, ${ }^{10, e}$ D. De Jesus Damiao, ${ }^{10}$ C. De Oliveira Martins, ${ }^{10}$ S. Fonseca De Souza, ${ }^{10}$ H. Malbouisson, ${ }^{10}$ D. Matos Figueiredo, ${ }^{10}$ M. Melo De Almeida, ${ }^{10}$ C. Mora Herrera,${ }^{10}$ L. Mundim, ${ }^{10}$ H. Nogima, ${ }^{10}$ W. L. Prado Da Silva,${ }^{10}$ L. J. Sanchez Rosas, ${ }^{10}$ A. Santoro, ${ }^{10}$ A. Sznajder, ${ }^{10}$ M. Thiel, ${ }^{10}$ E. J. Tonelli Manganote, ${ }^{10, d}$ F. Torres Da Silva De Araujo, ${ }^{10}$ A. Vilela Pereira, ${ }^{10}$ S. Ahuja, ${ }^{11 \mathrm{a}}$ C. A. Bernardes,${ }^{11 \mathrm{a}}$ L. Calligaris, ${ }^{1 \mathrm{a}}$ T. R. Fernandez Perez Tomei, ${ }^{11 \mathrm{a}}$ E. M. Gregores, ${ }^{11 \mathrm{a}, 11 \mathrm{~b}}$

P. G. Mercadante, ${ }^{11 \mathrm{a}, 1 \mathrm{~b}}$ S. F. Novaes, ${ }^{11 \mathrm{a}}$ Sandra S. Padula, ${ }^{11 \mathrm{a}}$ D. Romero Abad,${ }^{11 \mathrm{a}, 1 \mathrm{bb}}$ A. Aleksandrov, ${ }^{12}$ R. Hadjiiska, ${ }^{12}$ P. Iaydjiev, ${ }^{12}$ A. Marinov, ${ }^{12}$ M. Misheva, ${ }^{12}$ M. Rodozov, ${ }^{12}$ M. Shopova, ${ }^{12}$ G. Sultanov, ${ }^{12}$ A. Dimitrov, ${ }^{13}$ L. Litov, ${ }^{13}$ B. Pavlov, ${ }^{13}$ P. Petkov, ${ }^{13}$ W. Fang, ${ }^{14, f}$ X. Gao, ${ }^{14, f}$ L. Yuan, ${ }^{14}$ M. Ahmad, ${ }^{15}$ J. G. Bian, ${ }^{15}$ G. M. Chen, ${ }^{15}$ H. S. Chen, ${ }^{15}$ 
M. Chen, ${ }^{15}$ Y. Chen, ${ }^{15}$ C. H. Jiang, ${ }^{15}$ D. Leggat,${ }^{15}$ H. Liao, ${ }^{15}$ Z. Liu,${ }^{15}$ F. Romeo, ${ }^{15}$ S. M. Shaheen, ${ }^{15}$ A. Spiezia,${ }^{15}$ J. Tao,${ }^{15}$ C. Wang, ${ }^{15}$ Z. Wang, ${ }^{15}$ E. Yazgan, ${ }^{15}$ H. Zhang, ${ }^{15}$ J. Zhao, ${ }^{15}$ Y. Ban, ${ }^{16}$ G. Chen, ${ }^{16}$ A. Levin, ${ }^{16}$ J. Li, ${ }^{16}$ L. Li, ${ }^{16}$ Q. Li ${ }^{16}$ Y. Mao, ${ }^{16}$ S. J. Qian, ${ }^{16}$ D. Wang, ${ }^{16}$ Z. Xu, ${ }^{16}$ Y. Wang, ${ }^{17}$ C. Avila, ${ }^{18}$ A. Cabrera, ${ }^{18}$ C. A. Carrillo Montoya, ${ }^{18}$ L. F. Chaparro Sierra, ${ }^{18}$ C. Florez, ${ }^{18}$ C. F. González Hernández, ${ }^{18}$ M. A. Segura Delgado, ${ }^{18}$ B. Courbon, ${ }^{19}$ N. Godinovic, ${ }^{19}$ D. Lelas ${ }^{19}$ I. Puljak, ${ }^{19}$ T. Sculac, ${ }^{19}$ Z. Antunovic,${ }^{20}$ M. Kovac,${ }^{20}$ V. Brigljevic, ${ }^{21}$ D. Ferencek, ${ }^{21}$ K. Kadija, ${ }^{21}$ B. Mesic, ${ }^{21}$ A. Starodumov, ${ }^{21, g}$ T. Susa,${ }^{21}$ M. W. Ather, ${ }^{22}$ A. Attikis, ${ }^{22}$ M. Kolosova, ${ }^{22}$ G. Mavromanolakis, ${ }^{22}$ J. Mousa, ${ }^{22}$ C. Nicolaou, ${ }^{22}$ F. Ptochos,${ }^{22}$ P. A. Razis, ${ }^{22}$ H. Rykaczewski, ${ }^{22}$ M. Finger, ${ }^{23, h}$ M. Finger Jr., ${ }^{23, h}$ E. Ayala, ${ }^{24}$ E. Carrera Jarrin,${ }^{25}$ A. Mahrous, ${ }^{26, i}$ Y. Mohammed, ${ }^{26, j}$ E. Salama, ${ }^{26, k, 1}$ S. Bhowmik, ${ }^{27}$ A. Carvalho Antunes De Oliveira, ${ }^{27}$ R. K. Dewanjee, ${ }^{27}$ K. Ehataht, ${ }^{27}$ M. Kadastik, ${ }^{27}$ M. Raidal,${ }^{27}$ C. Veelken, ${ }^{27}$ P. Eerola,${ }^{28}$ H. Kirschenmann, ${ }^{28}$ J. Pekkanen, ${ }^{28}$ M. Voutilainen, ${ }^{28}$

J. Havukainen, ${ }^{29}$ J. K. Heikkilä, ${ }^{29}$ T. Järvinen, ${ }^{29}$ V. Karimäki, ${ }^{29}$ R. Kinnunen, ${ }^{29}$ T. Lampén, ${ }^{29}$ K. Lassila-Perini, ${ }^{29}$ S. Laurila, ${ }^{29}$ S. Lehti, ${ }^{29}$ T. Lindén, ${ }^{29}$ P. Luukka, ${ }^{29}$ T. Mäenpää, ${ }^{29}$ H. Siikonen, ${ }^{29}$ E. Tuominen, ${ }^{29}$ J. Tuominiemi, ${ }^{29}$ T. Tuuva, ${ }^{30}$ M. Besancon, ${ }^{31}$ F. Couderc, ${ }^{31}$ M. Dejardin, ${ }^{31}$ D. Denegri, ${ }^{31}$ J. L. Faure, ${ }^{31}$ F. Ferri, ${ }^{31}$ S. Ganjour, ${ }^{31}$ A. Givernaud, ${ }^{31}$ P. Gras,${ }^{31}$ G. Hamel de Monchenault,${ }^{31}$ P. Jarry,${ }^{31}$ C. Leloup,${ }^{31}$ E. Locci,${ }^{31}$ J. Malcles,${ }^{31}$ G. Negro, ${ }^{31}$ J. Rander, ${ }^{31}$ A. Rosowsky, ${ }^{31}$ M. Ö. Sahin, ${ }^{31}$ M. Titov, ${ }^{31}$ A. Abdulsalam, ${ }^{32, \mathrm{~m}}$ C. Amendola,${ }^{32}$ I. Antropov, ${ }^{32}$ F. Beaudette, ${ }^{32}$ P. Busson,${ }^{32}$ C. Charlot, ${ }^{32}$ R. Granier de Cassagnac, ${ }^{32}$ I. Kucher, ${ }^{32}$ S. Lisniak, ${ }^{32}$ A. Lobanov, ${ }^{32}$ J. Martin Blanco, ${ }^{32}$ M. Nguyen, ${ }^{32}$ C. Ochando, ${ }^{32}$ G. Ortona ${ }^{32}$ P. Pigard ${ }^{32}$ R. Salerno, ${ }^{32}$ J. B. Sauvan, ${ }^{32}$ Y. Sirois, ${ }^{32}$ A. G. Stahl Leiton, ${ }^{32}$ A. Zabi, ${ }^{32}$ A. Zghiche, ${ }^{32}$ J.-L. Agram, ${ }^{33, n}$ J. Andrea ${ }^{33}$ D. Bloch, ${ }^{33}$ J.-M. Brom, ${ }^{33}$ E. C. Chabert, ${ }^{33}$ V. Cherepanov, ${ }^{33}$ C. Collard ${ }^{33}$ E. Conte, ${ }^{33, n}$ J.-C. Fontaine, ${ }^{33, n}$ D. Gelé, ${ }^{33}$ U. Goerlach, ${ }^{33}$ M. Jansová, ${ }^{33}$ A.-C. Le Bihan, ${ }^{33}$ N. Tonon, ${ }^{33}$ P. Van Hove, ${ }^{33}$ S. Gadrat ${ }^{34}$ S. Beauceron, ${ }^{35}$ C. Bernet,${ }^{35}$ G. Boudoul, ${ }^{35}$ N. Chanon, ${ }^{35}$ R. Chierici, ${ }^{35}$ D. Contardo, ${ }^{35}$ P. Depasse, ${ }^{35}$ H. El Mamouni, ${ }^{35}$ J. Fay, ${ }^{35}$ L. Finco, ${ }^{35}$ S. Gascon, ${ }^{35}$ M. Gouzevitch, ${ }^{35}$ G. Grenier, ${ }^{35}$ B. Ille, ${ }^{35}$ F. Lagarde,${ }^{35}$ I. B. Laktineh, ${ }^{35}$ H. Lattaud, ${ }^{35}$ M. Lethuillier, ${ }^{35}$ L. Mirabito, ${ }^{35}$ A. L. Pequegnot, ${ }^{35}$ S. Perries, ${ }^{35}$ A. Popov, ${ }^{35,0}$ V. Sordini, ${ }^{35}$ M. Vander Donckt,,${ }^{35}$ S. Viret,${ }^{35}$ S. Zhang, ${ }^{35}$ T. Toriashvili, ${ }^{36, p}$ I. Bagaturia, ${ }^{37, q}$ C. Autermann, ${ }^{38}$ L. Feld, ${ }^{38}$ M. K. Kiesel, ${ }^{38}$ K. Klein, ${ }^{38}$ M. Lipinski, ${ }^{38}$ M. Preuten ${ }^{38}$ M. P. Rauch, ${ }^{38}$ C. Schomakers, ${ }^{38}$ J. Schulz,${ }^{38}$ M. Teroerde, ${ }^{38}$ B. Wittmer, ${ }^{38}$ V. Zhukov, ${ }^{38,0}$ A. Albert, ${ }^{39}$ D. Duchardt, ${ }^{39}$ M. Endres,${ }^{39}$ M. Erdmann, ${ }^{39}$ T. Esch, ${ }^{39}$ R. Fischer, ${ }^{39}$ S. Ghosh, ${ }^{39}$ A. Güth,${ }^{39}$ T. Hebbeker, ${ }^{39}$ C. Heidemann, ${ }^{39}$ K. Hoepfner, ${ }^{39}$ H. Keller, ${ }^{39}$ S. Knutzen, ${ }^{39}$ L. Mastrolorenzo, ${ }^{39}$ M. Merschmeyer, ${ }^{39}$ A. Meyer, ${ }^{39}$ P. Millet, ${ }^{39}$ S. Mukherjee, ${ }^{39}$ T. Pook, ${ }^{39}$ M. Radziej, ${ }^{39}$ H. Reithler, ${ }^{39}$ M. Rieger, ${ }^{39}$ F. Scheuch, ${ }^{39}$ A. Schmidt, ${ }^{39}$ D. Teyssier, ${ }^{39}$ G. Flügge, ${ }^{40}$

O. Hlushchenko, ${ }^{40}$ B. Kargoll, ${ }^{40}$ T. Kress, ${ }^{40}$ A. Künsken, ${ }^{40}$ T. Müller, ${ }^{40}$ A. Nehrkorn, ${ }^{40}$ A. Nowack, ${ }^{40}$ C. Pistone, ${ }^{40}$

O. Pooth, ${ }^{40}$ H. Sert, ${ }^{40}$ A. Stahl, ${ }^{40, r}$ M. Aldaya Martin,${ }^{41}$ T. Arndt,${ }^{41}$ C. Asawatangtrakuldee, ${ }^{41}$ I. Babounikau, ${ }^{41}$

K. Beernaert, ${ }^{41}$ O. Behnke, ${ }^{41}$ U. Behrens, ${ }^{41}$ A. Bermúdez Martínez ${ }^{41}$ D. Bertsche, ${ }^{41}$ A. A. Bin Anuar, ${ }^{41}$ K. Borras, ${ }^{41, s}$ V. Botta, ${ }^{41}$ A. Campbell, ${ }^{41}$ P. Connor, ${ }^{41}$ C. Contreras-Campana, ${ }^{41}$ F. Costanza, ${ }^{41}$ V. Danilov, ${ }^{41}$ A. De Wit, ${ }^{41}$ M. M. Defranchis, ${ }^{41}$ C. Diez Pardos, ${ }^{41}$ D. Domínguez Damiani, ${ }^{41}$ G. Eckerlin, ${ }^{41}$ T. Eichhorn, ${ }^{41}$ A. Elwood, ${ }^{41}$ E. Eren, ${ }^{41}$ E. Gallo, ${ }^{41, t}$ A. Geiser ${ }^{41}$ J. M. Grados Luyando, ${ }^{41}$ A. Grohsjean, ${ }^{41}$ P. Gunnellini,${ }^{41}$ M. Guthoff,${ }^{41}$ M. Haranko, ${ }^{41}$ A. Harb, ${ }^{41}$ J. Hauk ${ }^{41}$ H. Jung, ${ }^{41}$ M. Kasemann, ${ }^{41}$ J. Keaveney ${ }^{41}$ C. Kleinwort, ${ }^{41}$ J. Knolle, ${ }^{41}$ D. Krücker, ${ }^{41}$ W. Lange, ${ }^{41}$ A. Lelek, ${ }^{41}$ T. Lenz ${ }^{41}$ K. Lipka, ${ }^{41}$ W. Lohmann, ${ }^{41, u}$ R. Mankel,${ }^{41}$ I.-A. Melzer-Pellmann, ${ }^{41}$ A. B. Meyer, ${ }^{41}$ M. Meyer, ${ }^{41}$ M. Missiroli, ${ }^{41}$ G. Mittag, ${ }^{41}$ J. Mnich, ${ }^{41}$ V. Myronenko, ${ }^{41}$ S. K. Pflitsch, ${ }^{41}$ D. Pitzl, ${ }^{41}$ A. Raspereza, ${ }^{41}$ M. Savitskyi, ${ }^{41}$ P. Saxena, ${ }^{41}$ P. Schütze,${ }^{41}$ C. Schwanenberger,${ }^{41}$ R. Shevchenko, ${ }^{41}$ A. Singh,${ }^{41}$ N. Stefaniuk,${ }^{41}$ H. Tholen, ${ }^{41}$ O. Turkot, ${ }^{41}$ A. Vagnerini, ${ }^{41}$ G. P. Van Onsem, ${ }^{41}$ R. Walsh, ${ }^{41}$ Y. Wen, ${ }^{41}$ K. Wichmann, ${ }^{41}$ C. Wissing, ${ }^{41}$ O. Zenaiev, ${ }^{41}$ R. Aggleton, ${ }^{42}$ S. Bein, ${ }^{42}$

L. Benato, ${ }^{42}$ A. Benecke, ${ }^{42}$ V. Blobel, ${ }^{42}$ M. Centis Vignali, ${ }^{42}$ T. Dreyer, ${ }^{42}$ E. Garutti, ${ }^{42}$ D. Gonzalez, ${ }^{42}$ J. Haller, ${ }^{42}$ A. Hinzmann, ${ }^{42}$ A. Karavdina, ${ }^{42}$ G. Kasieczka, ${ }^{42}$ R. Klanner, ${ }^{42}$ R. Kogler, ${ }^{42}$ N. Kovalchuk, ${ }^{42}$ S. Kurz, ${ }^{42}$ V. Kutzner, ${ }^{42}$ J. Lange, ${ }^{42}$ D. Marconi,${ }^{42}$ J. Multhaup,${ }^{42}$ M. Niedziela, ${ }^{42}$ D. Nowatschin ${ }^{42}$ A. Perieanu, ${ }^{42}$ A. Reimers, ${ }^{42}$ O. Rieger, ${ }^{42}$ C. Scharf ${ }^{42}$ P. Schleper, ${ }^{42}$ S. Schumann, ${ }^{42}$ J. Schwandt, ${ }^{42}$ J. Sonneveld, ${ }^{42}$ H. Stadie ${ }^{42}$ G. Steinbrück, ${ }^{42}$ F. M. Stober, ${ }^{42}$ M. Stöver, ${ }^{42}$ D. Troendle, ${ }^{42}$ A. Vanhoefer, ${ }^{42}$ B. Vormwald, ${ }^{42}$ M. Akbiyik, ${ }^{43}$ C. Barth, ${ }^{43}$ M. Baselga, ${ }^{43}$ S. Baur, ${ }^{43}$ E. Butz,${ }^{43}$ R. Caspart, ${ }^{43}$ T. Chwalek, ${ }^{43}$ F. Colombo, ${ }^{43}$ W. De Boer, ${ }^{43}$ A. Dierlamm, ${ }^{43}$ N. Faltermann, ${ }^{43}$ B. Freund, ${ }^{43}$ M. Giffels, ${ }^{43}$ M. A. Harrendorf, ${ }^{43}$ F. Hartmann, ${ }^{43, r}$ S. M. Heindl, ${ }^{43}$ U. Husemann, ${ }^{43}$ F. Kassel,${ }^{43, r}$ I. Katkov, ${ }^{43,0}$ S. Kudella, ${ }^{43}$ H. Mildner, ${ }^{43}$ S. Mitra, ${ }^{43}$ M. U. Mozer, ${ }^{43}$ Th. Müller, ${ }^{43}$ M. Plagge, ${ }^{43}$ G. Quast, ${ }^{43}$ K. Rabbertz,${ }^{43}$ M. Schröder, ${ }^{43}$ I. Shvetsov, ${ }^{43}$ G. Sieber, ${ }^{43}$ H. J. Simonis, ${ }^{43}$ R. Ulrich, ${ }^{43}$ S. Wayand, ${ }^{43}$ M. Weber, ${ }^{43}$ T. Weiler, ${ }^{43}$ S. Williamson, ${ }^{43}$ C. Wöhrmann, ${ }^{43}$ R. Wolf, ${ }^{43}$ G. Anagnostou, ${ }^{44}$ G. Daskalakis, ${ }^{44}$ T. Geralis, ${ }^{44}$ A. Kyriakis, ${ }^{44}$ D. Loukas, ${ }^{44}$ G. Paspalaki, ${ }^{44}$ I. Topsis-Giotis, ${ }^{44}$ G. Karathanasis, ${ }^{45}$ S. Kesisoglou, ${ }^{45}$ P. Kontaxakis, ${ }^{45}$ A. Panagiotou, ${ }^{45}$ N. Saoulidou, ${ }^{45}$ E. Tziaferi ${ }^{45}$ K. Vellidis, ${ }^{45}$ K. Kousouris, ${ }^{46}$ I. Papakrivopoulos, ${ }^{46}$ G. Tsipolitis, ${ }^{46}$ I. Evangelou, ${ }^{47}$ C. Foudas, ${ }^{47}$ P. Gianneios, ${ }^{47}$ P. Katsoulis, ${ }^{47}$ 
P. Kokkas, ${ }^{47}$ S. Mallios,${ }^{47}$ N. Manthos,${ }^{47}$ I. Papadopoulos,${ }^{47}$ E. Paradas, ${ }^{47}$ J. Strologas, ${ }^{47}$ F. A. Triantis, ${ }^{47}$ D. Tsitsonis, ${ }^{47}$ M. Bartók, ${ }^{48, v}$ M. Csanad, ${ }^{48}$ N. Filipovic, ${ }^{48}$ P. Major, ${ }^{48}$ M. I. Nagy, ${ }^{48}$ G. Pasztor, ${ }^{48}$ O. Surányi, ${ }^{48}$ G. I. Veres,${ }^{48}$ G. Bencze,${ }^{49}$

C. Hajdu, ${ }^{49}$ D. Horvath, ${ }^{49, w}$ Á. Hunyadi, ${ }^{49}$ F. Sikler, ${ }^{49}$ T. Á. Vámi, ${ }^{49}$ V. Veszpremi, ${ }^{49}$ G. Vesztergombi, ${ }^{49, a, v}$ N. Beni, ${ }^{50}$

S. Czellar, ${ }^{50}$ J. Karancsi, ${ }^{50, x}$ A. Makovec, ${ }^{50}$ J. Molnar, ${ }^{50}$ Z. Szillasi, ${ }^{50}$ P. Raics,${ }^{51}$ Z. L. Trocsanyi, ${ }^{51}$ B. Ujvari, ${ }^{51}$

S. Choudhury, ${ }^{52}$ J. R. Komaragiri, ${ }^{52}$ P. C. Tiwari, ${ }^{52}$ S. Bahinipati, ${ }^{53, y}$ C. Kar, ${ }^{53}$ P. Mal, ${ }^{53}$ K. Mandal, ${ }^{53}$ A. Nayak,${ }^{53, z}$

D. K. Sahoo, ${ }^{53, y}$ S. K. Swain, ${ }^{53}$ S. Bansal, ${ }^{54}$ S. B. Beri, ${ }^{54}$ V. Bhatnagar, ${ }^{54}$ S. Chauhan, ${ }^{54}$ R. Chawla, ${ }^{54}$ N. Dhingra, ${ }^{54}$ R. Gupta, ${ }^{54}$ A. Kaur, ${ }^{54}$ A. Kaur, ${ }^{54}$ M. Kaur,${ }^{54}$ S. Kaur, ${ }^{54}$ R. Kumar, ${ }^{54}$ P. Kumari, ${ }^{54}$ M. Lohan, ${ }^{54}$ A. Mehta ${ }^{54}$ K. Sandeep,${ }^{54}$

S. Sharma,${ }^{54}$ J. B. Singh,${ }^{54}$ G. Walia ${ }^{54}$ A. Bhardwaj, ${ }^{55}$ B. C. Choudhary, ${ }^{55}$ R. B. Garg, ${ }^{55}$ M. Gola, ${ }^{55}$ S. Keshri, ${ }^{55}$ Ashok Kumar, ${ }^{55}$ S. Malhotra, ${ }^{55}$ M. Naimuddin, ${ }^{55}$ P. Priyanka,${ }^{55}$ K. Ranjan,${ }^{55}$ Aashaq Shah,${ }^{55}$ R. Sharma, ${ }^{55}$ R. Bhardwaj, ${ }^{56, a a}$ M. Bharti, ${ }^{56}$ R. Bhattacharya, ${ }^{56}$ S. Bhattacharya, ${ }^{56}$ U. Bhawandeep, ${ }^{56, a a}$ D. Bhowmik, ${ }^{56}$ S. Dey, ${ }^{56}$ S. Dutt, ${ }^{56, \text { aa }}$ S. Dutta,${ }^{56}$ S. Ghosh, ${ }^{56}$ K. Mondal, ${ }^{56}$ S. Nandan, ${ }^{56}$ A. Purohit, ${ }^{56}$ P. K. Rout, ${ }^{56}$ A. Roy, ${ }^{56}$ S. Roy Chowdhury,${ }^{56}$ S. Sarkar,${ }^{56}$ M. Sharan, ${ }^{56}$

B. Singh, ${ }^{56}$ S. Thakur, ${ }^{56, a a}$ P. K. Behera,${ }^{57}$ R. Chudasama, ${ }^{58}$ D. Dutta, ${ }^{58}$ V. Jha, ${ }^{58}$ V. Kumar, ${ }^{58}$ P. K. Netrakanti, ${ }^{58}$ L. M. Pant,${ }^{58}$ P. Shukla, ${ }^{58}$ T. Aziz, ${ }^{59}$ M. A. Bhat, ${ }^{59}$ S. Dugad,${ }^{59}$ G. B. Mohanty, ${ }^{59}$ N. Sur, ${ }^{59}$ B. Sutar,${ }^{59}$

Ravindra Kumar Verma, ${ }^{50}$ S. Banerjee, ${ }^{60}$ S. Bhattacharya,${ }^{60}$ S. Chatterjee,${ }^{60}$ P. Das,${ }^{60}$ M. Guchait, ${ }^{60}$ Sa. Jain, ${ }^{60}$

S. Karmakar, ${ }^{60}$ S. Kumar, ${ }^{60}$ M. Maity, ${ }^{60, b b}$ G. Majumder ${ }^{60}$ K. Mazumdar, ${ }^{60}$ N. Sahoo, ${ }^{60}$ T. Sarkar, ${ }^{60, b b}$ S. Chauhan, ${ }^{61}$

S. Dube, ${ }^{61}$ V. Hegde ${ }^{61}$ A. Kapoor,${ }^{61}$ K. Kothekar, ${ }^{61}$ S. Pandey, ${ }^{61}$ A. Rane, ${ }^{61}$ S. Sharma, ${ }^{61}$ S. Chenarani, ${ }^{62, c c}$

E. Eskandari Tadavani, ${ }^{62}$ S. M. Etesami, ${ }^{62, c c}$ M. Khakzad ${ }^{62}$ M. Mohammadi Najafabadi, ${ }^{62}$ M. Naseri, ${ }^{62}$

F. Rezaei Hosseinabadi, ${ }^{62}$ B. Safarzadeh, ${ }^{62, d d}$ M. Zeinali, ${ }^{62}$ M. Felcini, ${ }^{63}$ M. Grunewald, ${ }^{63}$ M. Abbrescia, ${ }^{64 a, 64 b}$

C. Calabria, ${ }^{64 a, 64 b}$ A. Colaleo, ${ }^{64 a}$ D. Creanza, ${ }^{64 a, 64 c}$ L. Cristella,${ }^{64 a, 64 b}$ N. De Filippis, ${ }^{64 a, 64 c}$ M. De Palma,${ }^{64 a, 64 b}$

A. Di Florio, ${ }^{64 a, 64 b}$ F. Errico ${ }^{64 a, 64 b}$ L. Fiore, ${ }^{64 a}$ A. Gelmi, ${ }^{64 a, 64 b}$ G. Iaselli, ${ }^{64 a, 64 c}$ S. Lezki, ${ }^{64 a, 64 b}$ G. Maggi, ${ }^{64 a, 64 c}$ M. Maggi, ${ }^{64 a}$

G. Miniello, ${ }^{64,64 b}$ S. My, ${ }^{64 a, 64 b}$ S. Nuzzo, ${ }^{64 a, 64 b}$ A. Pompili, ${ }^{64 a, 64 b}$ G. Pugliese ${ }^{64 a, 64 c}$ R. Radogna, ${ }^{64 a}$ A. Ranieri, ${ }^{64 a}$

G. Selvaggi, ${ }^{64 a, 64 b}$ A. Sharma, ${ }^{64 a}$ L. Silvestris, ${ }^{64 a, r}$ R. Venditti, ${ }^{64 a}$ P. Verwilligen, ${ }^{64 a}$ G. Zito, ${ }^{64 a}$ G. Abbiendi, ${ }^{65 a}$

C. Battilana, ${ }^{65 a, 65 b}$ D. Bonacorsi, ${ }^{65 a, 65 b}$ L. Borgonovi ${ }^{65 a, 65 b}$ S. Braibant-Giacomelli, ${ }^{65 a, 65 b}$ R. Campanini, ${ }^{65 a, 65 b}$

P. Capiluppi, ${ }^{65 a, 65 b}$ A. Castro, ${ }^{65 a, 65 b}$ F. R. Cavallo ${ }^{65 a}$ S. S. Chhibra,${ }^{65 a, 65 b}$ C. Ciocca ${ }^{65 a}$ G. Codispoti, ${ }^{65 a, 65 b}$ M. Cuffiani ${ }^{65 a, 65 b}$

G. M. Dallavalle, ${ }^{65 a}$ F. Fabbri, ${ }^{65 a}$ A. Fanfani, ${ }^{65,65 b}$ P. Giacomelli, ${ }^{65 a}$ C. Grandi, ${ }^{65 a}$ L. Guiducci, ${ }^{65 a, 65 b}$ F. Iemmi, ${ }^{65 a, 65 b}$

S. Marcellini, ${ }^{65 a}$ G. Masetti, ${ }^{65 a}$ A. Montanari, ${ }^{65 a}$ F. L. Navarria ${ }^{65 a, 65 b}$ A. Perrotta, ${ }^{65 a}$ F. Primavera, ${ }^{65 a, 65 b, r}$ A. M. Rossi, ${ }^{65 a, 65 b}$

T. Rovelli, ${ }^{65 a, 65 b}$ G. P. Siroli, ${ }^{65 a, 65 b}$ N. Tosi, ${ }^{65 a}$ S. Albergo,${ }^{66 a, 66 b}$ A. Di Mattia ${ }^{66 a}$ R. Potenza, ${ }^{66 a, 66 b}$ A. Tricomi, ${ }^{66 a, 66 b}$

C. Tuve ${ }^{66 a, 66 b}$ G. Barbagli, ${ }^{67 a}$ K. Chatterjee, ${ }^{67 a, 67 b}$ V. Ciulli, ${ }^{67 a, 67 b}$ C. Civinini, ${ }^{67 a}$ R. D'Alessandro, ${ }^{67 a, 67 b}$ E. Focardi, ${ }^{67 a, 67 b}$

G. Latino, ${ }^{67 a}$ P. Lenzi, ${ }^{67 a, 67 b}$ M. Meschini, ${ }^{67 a}$ S. Paoletti, ${ }^{67 a}$ L. Russo, ${ }^{67 a, e e}$ G. Sguazzoni, ${ }^{67 a}$ D. Strom, ${ }^{67 a}$ L. Viliani, ${ }^{67 a}$

L. Benussi ${ }^{68}$ S. Bianco, ${ }^{68}$ F. Fabbri, ${ }^{68}$ D. Piccolo,${ }^{68}$ F. Ferro, ${ }^{69 a}$ F. Ravera,${ }^{69 a, 69 b}$ E. Robutti ${ }^{69 a}$ S. Tosi,${ }^{69 a, 69 b}$ A. Benaglia, ${ }^{70 a}$

A. Beschi, ${ }^{70 a, 70 b}$ L. Brianza, ${ }^{70 a, 70 b}$ F. Brivio, ${ }^{70 a, 70 b}$ V. Ciriolo, ${ }^{70 a, 70 b, r}$ S. Di Guida ${ }^{70 a, 70 b, r}$ M. E. Dinardo, ${ }^{70 a, 70 b}$

S. Fiorendi, ${ }^{70 a, 70 b}$ S. Gennai, ${ }^{70 a}$ A. Ghezzi, ${ }^{70 a, 70 b}$ P. Govoni, ${ }^{70 a, 70 b}$ M. Malberti, ${ }^{70 a, 70 b}$ S. Malvezzi, ${ }^{70 a}$ A. Massironi, ${ }^{70 a, 70 b}$

D. Menasce, ${ }^{70 a}$ L. Moroni, ${ }^{70 a}$ M. Paganoni, ${ }^{70 a, 70 b}$ D. Pedrini, ${ }^{70 a}$ S. Ragazzi,${ }^{70 a, 70 b}$ T. Tabarelli de Fatis, ${ }^{70 a, 70 b}$

S. Buontempo, ${ }^{71 \mathrm{a}}$ N. Cavallo, ${ }^{71 a, 71 \mathrm{c}}$ A. Di Crescenzo, ${ }^{71 \mathrm{a}, 71 \mathrm{~b}}$ F. Fabozzi, ${ }^{71 \mathrm{a}, 71 \mathrm{c}}$ F. Fienga, ${ }^{71 \mathrm{a}}$ G. Galati, ${ }^{71 \mathrm{a}}$ A. O. M. Iorio, ${ }^{71 \mathrm{a}, 71 \mathrm{~b}}$ W. A. Khan, ${ }^{71 \mathrm{a}}$ L. Lista $^{71 \mathrm{a}}$ S. Meola, ${ }^{71 \mathrm{a}, 71 \mathrm{~d}, \mathrm{r}}$ P. Paolucci, ${ }^{71 \mathrm{a}, \mathrm{r}}$ C. Sciacca, ${ }^{71 \mathrm{a}, 71 \mathrm{~b}}$ E. Voevodina,${ }^{71 \mathrm{a}, 71 \mathrm{~b}}$ P. Azzi, ${ }^{72 a}$ N. Bacchetta, ${ }^{72 a}$ D. Bisello, ${ }^{72 a, 72 b}$ A. Boletti, ${ }^{72 a, 72 b}$ A. Bragagnolo, ${ }^{72 a}$ R. Carlin, ${ }^{72 a, 72 b}$ P. Checchia, ${ }^{72 a}$ M. Dall'Osso, ${ }^{72 a, 72 b}$

P. De Castro Manzano, ${ }^{72 a}$ T. Dorigo, ${ }^{72 a}$ F. Gasparini, ${ }^{72 a, 72 b}$ U. Gasparini, ${ }^{72 a, 72 b}$ S. Lacaprara, ${ }^{72 a}$ P. Lujan, ${ }^{72 a}$

M. Margoni, ${ }^{72 a, 72 b}$ A. T. Meneguzzo,${ }^{72 a, 72 b}$ N. Pozzobon, ${ }^{72 a, 72 b}$ P. Ronchese, ${ }^{72 a, 72 b}$ R. Rossin, ${ }^{72 a, 72 b}$ F. Simonetto, ${ }^{72 a, 72 b}$ A. Tiko, ${ }^{72 a}$ E. Torassa, ${ }^{72 a}$ S. Ventura, ${ }^{72 a}$ M. Zanetti, ${ }^{72 a, 72 b}$ P. Zotto, ${ }^{72 a, 72 b}$ G. Zumerle, ${ }^{72 a, 72 b}$ A. Braghieri, ${ }^{73 a}$ A. Magnani, ${ }^{73 a}$ P. Montagna, ${ }^{73 a, 73 b}$ S. P. Ratti, ${ }^{73 a, 73 b}$ V. Re,${ }^{73 a}$ M. Ressegotti, ${ }^{73 a, 73 b}$ C. Riccardi, ${ }^{73 a, 73 b}$ P. Salvini, ${ }^{73 a}$ I. Vai, ${ }^{73 a, 73 b}$ P. Vitulo ${ }^{73 a, 73 b}$ L. Alunni Solestizi, ${ }^{74 a, 74 b}$ M. Biasini, ${ }^{74 a, 74 b}$ G. M. Bilei, ${ }^{74 a}$ C. Cecchi, ${ }^{74 a, 74 b}$ D. Ciangottini, ${ }^{74 a, 74 b}$ L. Fanò, ${ }^{74 a, 74 b}$ P. Lariccia, ${ }^{74 a, 74 b}$ E. Manoni, ${ }^{74 a}$ G. Mantovani, ${ }^{74 a, 74 b}$ V. Mariani, ${ }^{74 a, 74 b}$ M. Menichelli, ${ }^{74 a}$ A. Rossi, ${ }^{74 a, 74 b}$ A. Santocchia, ${ }^{74 a, 74 b}$ D. Spiga ${ }^{74 a}$ K. Androsov, ${ }^{75 a}$ P. Azzurri, ${ }^{75 a}$ G. Bagliesi, ${ }^{75 a}$ L. Bianchini, ${ }^{75 a}$ T. Boccali, ${ }^{75 a}$ L. Borrello, ${ }^{75 a}$ R. Castaldi, ${ }^{75 a}$

M. A. Ciocci, ${ }^{75 a}, 75 b$ R. Dell'Orso, ${ }^{75 a}$ G. Fedi, ${ }^{75 a}$ F. Fiori, ${ }^{75 a, 75 c}$ L. Giannini, ${ }^{75 a, 75 c}$ A. Giassi, ${ }^{75 a}$ M. T. Grippo, ${ }^{75 a}$

F. Ligabue ${ }^{75 a, 75 c}$ E. Manca ${ }^{75 a, 75 c}$ G. Mandorli, ${ }^{75 a, 75 c}$ A. Messineo,${ }^{75 a, 75 b}$ F. Palla, ${ }^{75 a}$ A. Rizzi, ${ }^{75 a, 75 b}$ P. Spagnolo, ${ }^{75 a}$ R. Tenchini, ${ }^{75 a}$ G. Tonelli, ${ }^{75 a}{ }^{75 b}$ A. Venturi, ${ }^{75 a}$ P. G. Verdini, ${ }^{75 a}$ L. Barone,${ }^{76 a, 76 b}$ F. Cavallari, ${ }^{76 a}$ M. Cipriani, ${ }^{76 a, 76 b}$ N. Daci, ${ }^{76 a}$ D. Del Re ${ }^{76 a, 76 b}$ E. Di Marco, ${ }^{76 a, 76 b}$ M. Diemoz,${ }^{76 a}$ S. Gelli, ${ }^{76 a, 76 b}$ E. Longo, ${ }^{76 a, 76 b}$ B. Marzocchi ${ }^{76 a, 76 b}$ P. Meridiani, ${ }^{76 a}$ G. Organtini, ${ }^{76 a, 76 b}$ F. Pandolfi, ${ }^{76 \mathrm{a}}$ R. Paramatti, ${ }^{76 a, 76 \mathrm{~b}}$ F. Preiato, ${ }^{76 a, 76 \mathrm{~b}}$ S. Rahatlou, ${ }^{76 a, 76 \mathrm{~b}}$ C. Rovelli, ${ }^{76 \mathrm{a}}$ F. Santanastasio, ${ }^{76 \mathrm{a}, 76 \mathrm{~b}}$ N. Amapane, ${ }^{77 a, 77 b}$ R. Arcidiacono, ${ }^{77 a, 77 c}$ S. Argiro, ${ }^{77,77 b}$ M. Arneodo, ${ }^{77,77 c}$ N. Bartosik, ${ }^{77 a}$ R. Bellan, ${ }^{77,77 b}$ C. Biino, ${ }^{77 a}$ 
N. Cartiglia, ${ }^{77 a}$ F. Cenna, ${ }^{77 a, 77 b}$ S. Cometti, ${ }^{77 a}$ M. Costa,${ }^{77 a, 77 b}$ R. Covarelli, ${ }^{77 a, 77 b}$ N. Demaria, ${ }^{77 a}$ B. Kiani, ${ }^{77 a, 77 b}$ C. Mariotti, ${ }^{77 a}$ S. Maselli, ${ }^{77 a}$ E. Migliore,${ }^{77 a, 77 b}$ V. Monaco, ${ }^{77 a, 77 b}$ E. Monteil, ${ }^{77 a, 77 b}$ M. Monteno, ${ }^{77 a}$ M. M. Obertino, ${ }^{77 a, 77 b}$ L. Pacher, ${ }^{77,77 b}$ N. Pastrone, ${ }^{77 a}$ M. Pelliccioni, ${ }^{77 a}$ G. L. Pinna Angioni, ${ }^{77 a, 77 b}$ A. Romero, ${ }^{77 a, 77 b}$ M. Ruspa,${ }^{77 a, 77 c}$ R. Sacchi, ${ }^{77 a, 77 b}$ K. Shchelina, ${ }^{77,77 b}$ V. Sola, ${ }^{77 a}$ A. Solano, ${ }^{77 a, 77 b}$ D. Soldi, ${ }^{77 a}$ A. Staiano, ${ }^{77 a}$ S. Belforte, ${ }^{78 a}$ V. Candelise, ${ }^{78 a, 78 b}$ M. Casarsa, ${ }^{78 a}$ F. Cossutti, ${ }^{78 a}$ G. Della Ricca, ${ }^{78 a, 78 b}$ F. Vazzoler, ${ }^{78 a, 78 b}$ A. Zanetti, ${ }^{78 a}$ D. H. Kim, ${ }^{79}$ G. N. Kim, ${ }^{79}$ M. S. Kim, ${ }^{79}$ J. Lee, ${ }^{79}$ S. Lee, ${ }^{79}$ S. W. Lee, ${ }^{79}$ C. S. Moon, ${ }^{79}$ Y. D. Oh, ${ }^{79}$ S. Sekmen, ${ }^{79}$ D. C. Son, ${ }^{79}$ Y. C. Yang,${ }^{79}$ H. Kim, ${ }^{80}$ D. H. Moon, ${ }^{80}$ G. Oh, ${ }^{80}$ J. Goh ${ }^{81}$ T. J. Kim, ${ }^{81}$ S. Cho, ${ }^{82}$ S. Choi, ${ }^{82}$ Y. Go, ${ }^{82}$ D. Gyun, ${ }^{82}$ S. Ha, ${ }^{82}$ B. Hong, ${ }^{82}$ Y. Jo, ${ }^{82}$ K. Lee, ${ }^{82}$ K. S. Lee, ${ }^{82}$ S. Lee, ${ }^{82}$ J. Lim, ${ }^{82}$ S. K. Park, ${ }^{82}$ Y. Roh,${ }^{82}$ H. S. Kim, ${ }^{83}$ J. Almond, ${ }^{84}$ J. Kim, ${ }^{84}$ J. S. Kim, ${ }^{84}$ H. Lee, ${ }^{84}$ K. Lee, ${ }^{84}$ K. Nam, ${ }^{84}$ S. B. Oh ${ }^{84}$ B. C. Radburn-Smith, ${ }^{84}$ S. h. Seo ${ }^{84}$ U. K. Yang, ${ }^{84}$ H. D. Yoo, ${ }^{84}$ G. B. Yu ${ }^{84}$ D. Jeon,${ }^{85}$ H. Kim, ${ }^{85}$ J. H. Kim, ${ }^{85}$ J. S. H. Lee, ${ }^{85}$ I. C. Park, ${ }^{85}$ Y. Choi, ${ }^{86}$ C. Hwang,${ }^{86}$ J. Lee,${ }^{86}$ I. Yu, ${ }^{86}$ V. Dudenas, ${ }^{87}$ A. Juodagalvis, ${ }^{87}$ J. Vaitkus, ${ }^{87}$ I. Ahmed ${ }^{88}$ Z. A. Ibrahim, ${ }^{88}$ M. A. B. Md Ali, ${ }^{88, f f}$ F. Mohamad Idris, ${ }^{88, g g}$ W. A. T. Wan Abdullah, ${ }^{88}$ M. N. Yusli, ${ }^{88}$ Z. Zolkapli, ${ }^{88}$

A. Castaneda Hernandez, ${ }^{89}$ J. A. Murillo Quijada,${ }^{89}$ H. Castilla-Valdez,${ }^{90}$ E. De La Cruz-Burelo, ${ }^{90}$ M. C. Duran-Osuna, ${ }^{90}$ I. Heredia-De La Cruz, ${ }^{90, \text { hh }}$ R. Lopez-Fernandez, ${ }^{90}$ J. Mejia Guisao, ${ }^{90}$ R. I. Rabadan-Trejo, ${ }^{90}$ G. Ramirez-Sanchez, ${ }^{90}$ R. Reyes-Almanza, ${ }^{90}$ A. Sanchez-Hernandez ${ }^{90}$ S. Carrillo Moreno, ${ }^{91}$ C. Oropeza Barrera, ${ }^{91}$ F. Vazquez Valencia ${ }^{91}$ J. Eysermans, ${ }^{92}$ I. Pedraza, ${ }^{92}$ H. A. Salazar Ibarguen, ${ }^{92}$ C. Uribe Estrada,${ }^{92}$ A. Morelos Pineda,${ }^{93}$ D. Krofcheck, ${ }^{94}$ S. Bheesette, ${ }^{95}$ P. H. Butler, ${ }^{95}$ A. Ahmad, ${ }^{96}$ M. Ahmad, ${ }^{96}$ M. I. Asghar, ${ }^{96}$ Q. Hassan, ${ }^{96}$ H. R. Hoorani, ${ }^{96}$ A. Saddique, ${ }^{96}$ M. A. Shah, ${ }^{96}$ M. Shoaib, ${ }^{96}$ M. Waqas,${ }^{96}$ H. Bialkowska, ${ }^{97}$ M. Bluj,${ }^{97}$ B. Boimska,${ }^{97}$ T. Frueboes,${ }^{97}$ M. Górski, ${ }^{97}$ M. Kazana, ${ }^{97}$ K. Nawrocki ${ }^{97}$ M. Szleper, ${ }^{97}$ P. Traczyk, ${ }^{97}$ P. Zalewski, ${ }^{97}$ K. Bunkowski, ${ }^{98}$ A. Byszuk,${ }^{98, i i}$ K. Doroba, ${ }^{98}$ A. Kalinowski, ${ }^{98}$ M. Konecki, ${ }^{98}$ J. Krolikowski, ${ }^{98}$ M. Misiura, ${ }^{98}$ M. Olszewski, ${ }^{98}$ A. Pyskir, ${ }^{98}$ M. Walczak,${ }^{98}$ P. Bargassa,${ }^{99}$ C. Beirão Da Cruz E Silva, ${ }^{99}$ A. Di Francesco,${ }^{99}$ P. Faccioli, ${ }^{99}$ B. Galinhas,${ }^{99}$ M. Gallinaro, ${ }^{99}$ J. Hollar, ${ }^{99}$ N. Leonardo, ${ }^{99}$ L. Lloret Iglesias, ${ }^{99}$ M. V. Nemallapudi ${ }^{99}$ J. Seixas, ${ }^{99}$ G. Strong, ${ }^{99}$ O. Toldaiev, ${ }^{99}$ D. Vadruccio, ${ }^{99}$ J. Varela, ${ }^{99}$

A. Baginyan, ${ }^{100}$ I. Golutvin, ${ }^{100}$ V. Karjavin, ${ }^{100}$ I. Kashunin, ${ }^{100}$ V. Korenkov, ${ }^{100}$ G. Kozlov, ${ }^{100}$ A. Lanev, ${ }^{100}$ A. Malakhov, ${ }^{100}$ V. Matveev, ${ }^{100, j j, k k}$ V. V. Mitsyn, ${ }^{100}$ P. Moisenz, ${ }^{100}$ V. Palichik, ${ }^{100}$ V. Perelygin, ${ }^{100}$ S. Shmatov, ${ }^{100}$ N. Skatchkov, ${ }^{100}$ V. Smirnov, ${ }^{100}$ V. Trofimov, ${ }^{100}$ A. Zarubin, ${ }^{100}$ V. Zhiltsov, ${ }^{100}$ V. Golovtsov, ${ }^{101}$ Y. Ivanov, ${ }^{101}$ V. Kim, ${ }^{101,11}$ E. Kuznetsova, ${ }^{101, m m}$ P. Levchenko, ${ }^{101}$ V. Murzin, ${ }^{101}$ V. Oreshkin, ${ }^{101}$ I. Smirnov, ${ }^{101}$ D. Sosnov, ${ }^{101}$ V. Sulimov, ${ }^{101}$ L. Uvarov, ${ }^{101}$ S. Vavilov, ${ }^{101}$ A. Vorobyev, ${ }^{101}$ Yu. Andreev, ${ }^{102}$ A. Dermenev, ${ }^{102}$ S. Gninenko, ${ }^{102}$ N. Golubev, ${ }^{102}$ A. Karneyeu, ${ }^{102}$ M. Kirsanov, ${ }^{102}$ N. Krasnikov, ${ }^{102}$ A. Pashenkov, ${ }^{102}$ D. Tlisov, ${ }^{102}$ A. Toropin, ${ }^{102}$ V. Epshteyn,${ }^{103}$ V. Gavrilov, ${ }^{103}$ N. Lychkovskaya, ${ }^{103}$ V. Popov, ${ }^{103}$ I. Pozdnyakov, ${ }^{103}$ G. Safronov, ${ }^{103}$ A. Spiridonov, ${ }^{103}$ A. Stepennov, ${ }^{103}$ V. Stolin, ${ }^{103}$ M. Toms,${ }^{103}$ E. Vlasov, ${ }^{103}$ A. Zhokin, ${ }^{103}$ T. Aushev, ${ }^{104}$ R. Chistov, ${ }^{105, n n}$ M. Danilov, ${ }^{105, n n}$ P. Parygin, ${ }^{105}$ D. Philippov, ${ }^{105}$ S. Polikarpov, ${ }^{105, n n}$ E. Tarkovskii, ${ }^{105}$ V. Andreev, ${ }^{106}$ M. Azarkin, ${ }^{106, k k}$ I. Dremin,${ }^{106, k \mathrm{kk}}$ M. Kirakosyan, ${ }^{106, \mathrm{kk}}$ S. V. Rusakov, ${ }^{106}$ A. Terkulov, ${ }^{106}$ A. Baskakov, ${ }^{107}$ A. Belyaev, ${ }^{107}$ E. Boos, ${ }^{107}$ M. Dubinin, ${ }^{107,00}$ L. Dudko, ${ }^{107}$ A. Ershov, ${ }^{107}$ A. Gribushin, ${ }^{107}$ V. Klyukhin, ${ }^{107}$ O. Kodolova, ${ }^{107}$ I. Lokhtin, ${ }^{107}$ I. Miagkov, ${ }^{107}$ S. Obraztsov, ${ }^{107}$ S. Petrushanko, ${ }^{107}$ V. Savrin, ${ }^{107}$ A. Snigirev, ${ }^{107}$ V. Blinov, ${ }^{108, p p}$

T. Dimova, ${ }^{108, p p}$ L. Kardapoltsev, ${ }^{108, p p}$ D. Shtol, ${ }^{108, p p}$ Y. Skovpen, ${ }^{108, p p}$ I. Azhgirey, ${ }^{109}$ I. Bayshev, ${ }^{109}$ S. Bitioukov, ${ }^{109}$

D. Elumakhov, ${ }^{109}$ A. Godizov, ${ }^{109}$ V. Kachanov, ${ }^{109}$ A. Kalinin, ${ }^{109}$ D. Konstantinov, ${ }^{109}$ P. Mandrik, ${ }^{109}$ V. Petrov, ${ }^{109}$ R. Ryutin, ${ }^{109}$ S. Slabospitskii, ${ }^{109}$ A. Sobol, ${ }^{109}$ S. Troshin,${ }^{109}$ N. Tyurin, ${ }^{109}$ A. Uzunian, ${ }^{109}$ A. Volkov, ${ }^{109}$ A. Babaev, ${ }^{110}$ S. Baidali, ${ }^{110}$ P. Adzic, ${ }^{11, q q}$ P. Cirkovic, ${ }^{111}$ D. Devetak, ${ }^{111}$ M. Dordevic, ${ }^{111}$ J. Milosevic, ${ }^{111}$ J. Alcaraz Maestre, ${ }^{112}$ A. Álvarez Fernández, ${ }^{112}$ I. Bachiller ${ }^{112}$ M. Barrio Luna, ${ }^{112}$ J. A. Brochero Cifuentes, ${ }^{112}$ M. Cerrada ${ }^{112}$ N. Colino, ${ }^{112}$ B. De La Cruz, ${ }^{112}$ A. Delgado Peris, ${ }^{112}$ C. Fernandez Bedoya ${ }^{112}$ J. P. Fernández Ramos, ${ }^{112}$ J. Flix, ${ }^{112}$ M. C. Fouz, ${ }^{112}$ O. Gonzalez Lopez, ${ }^{112}$ S. Goy Lopez, ${ }^{112}$ J. M. Hernandez, ${ }^{112}$ M. I. Josa, ${ }^{112}$ D. Moran, ${ }^{112}$ A. Pérez-Calero Yzquierdo, ${ }^{112}$ J. Puerta Pelayo, ${ }^{112}$ I. Redondo, ${ }^{112}$ L. Romero, ${ }^{112}$ M. S. Soares,${ }^{112}$ A. Triossi, ${ }^{112}$ C. Albajar, ${ }^{113}$ J. F. de Trocóniz, ${ }^{113}$ J. Cuevas, ${ }^{114}$ C. Erice, ${ }^{114}$ J. Fernandez Menendez, ${ }^{114}$ S. Folgueras, ${ }^{114}$ I. Gonzalez Caballero, ${ }^{114}$ J. R. González Fernández, ${ }^{114}$ E. Palencia Cortezon, ${ }^{114}$ V. Rodríguez Bouza, ${ }^{114}$ S. Sanchez Cruz, ${ }^{114}$ P. Vischia, ${ }^{114}$ J. M. Vizan Garcia, ${ }^{114}$ I. J. Cabrillo, ${ }^{115}$

A. Calderon, ${ }^{115}$ B. Chazin Quero, ${ }^{115}$ J. Duarte Campderros, ${ }^{115}$ M. Fernandez, ${ }^{115}$ P. J. Fernández Manteca, ${ }^{115}$

A. García Alonso, ${ }^{115}$ J. Garcia-Ferrero, ${ }^{115}$ G. Gomez, ${ }^{115}$ A. Lopez Virto, ${ }^{115}$ J. Marco, ${ }^{115}$ C. Martinez Rivero, ${ }^{115}$ P. Martinez Ruiz del Arbol, ${ }^{115}$ F. Matorras, ${ }^{115}$ J. Piedra Gomez, ${ }^{115}$ C. Prieels, ${ }^{115}$ T. Rodrigo, ${ }^{115}$ A. Ruiz-Jimeno, ${ }^{115}$ L. Scodellaro, ${ }^{115}$ N. Trevisani, ${ }^{115}$ I. Vila, ${ }^{115}$ R. Vilar Cortabitarte, ${ }^{115}$ D. Abbaneo, ${ }^{116}$ B. Akgun, ${ }^{116}$ E. Auffray,${ }^{116}$ P. Baillon, ${ }^{116}$ A. H. Ball, ${ }^{116}$ D. Barney, ${ }^{116}$ J. Bendavid, ${ }^{116}$ M. Bianco, ${ }^{116}$ A. Bocci, ${ }^{116}$ C. Botta, ${ }^{116}$ T. Camporesi, ${ }^{116}$ M. Cepeda, ${ }^{116}$ G. Cerminara, ${ }^{116}$ E. Chapon, ${ }^{116}$ Y. Chen, ${ }^{116}$ G. Cucciati, ${ }^{116}$ D. d'Enterria, ${ }^{116}$ A. Dabrowski, ${ }^{116}$ V. Daponte, ${ }^{116}$ A. David, ${ }^{116}$ A. De Roeck, ${ }^{116}$ N. Deelen, ${ }^{116}$ M. Dobson, ${ }^{116}$ T. du Pree, ${ }^{116}$ M. Dünser, ${ }^{116}$ N. Dupont, ${ }^{116}$ A. Elliott-Peisert, ${ }^{116}$ 
P. Everaerts, ${ }^{116}$ F. Fallavollita, ${ }^{116, r \mathrm{r}}$ D. Fasanella, ${ }^{116}$ G. Franzoni, ${ }^{116}$ J. Fulcher, ${ }^{116}$ W. Funk, ${ }^{116}$ D. Gigi, ${ }^{116}$ A. Gilbert, ${ }^{116}$ K. Gill, ${ }^{116}$ F. Glege, ${ }^{116}$ M. Guilbaud, ${ }^{116}$ D. Gulhan, ${ }^{16}$ J. Hegeman, ${ }^{116}$ V. Innocente, ${ }^{116}$ A. Jafari, ${ }^{116}$ P. Janot, ${ }^{116}$ O. Karacheban, ${ }^{16, u}$ J. Kieseler, ${ }^{116}$ A. Kornmayer, ${ }^{116}$ M. Krammer, ${ }^{116, b}$ C. Lange, ${ }^{116}$ P. Lecoq, ${ }^{116}$ C. Lourenço, ${ }^{116}$ L. Malgeri, ${ }^{116}$ M. Mannelli, ${ }^{116}$ F. Meijers, ${ }^{116}$ J. A. Merlin, ${ }^{116}$ S. Mersi, ${ }^{116}$ E. Meschi, ${ }^{116}$ P. Milenovic, ${ }^{116, s s}$ F. Moortgat, ${ }^{116}$ M. Mulders, ${ }^{16}$ J. Ngadiuba, ${ }^{116}$ S. Orfanelli, ${ }^{16}$ L. Orsini, ${ }^{16}$ F. Pantaleo, ${ }^{116, \mathrm{r}}$ L. Pape, ${ }^{116}$ E. Perez, ${ }^{116}$ M. Peruzzi, ${ }^{116}$ A. Petrilli, ${ }^{116}$ G. Petrucciani, ${ }^{116}$ A. Pfeiffer, ${ }^{116}$ M. Pierini, ${ }^{116}$ F. M. Pitters, ${ }^{116}$ D. Rabady, ${ }^{16}$ A. Racz, ${ }^{116}$ T. Reis, ${ }^{116}$ G. Rolandi, ${ }^{116, t \mathrm{tt}}$ M. Rovere, ${ }^{116}$ H. Sakulin, ${ }^{116}$ C. Schäfer, ${ }^{116}$ C. Schwick,${ }^{116}$ M. Seidel,${ }^{116}$ M. Selvaggi, ${ }^{116}$ A. Sharma, ${ }^{116}$ P. Silva, ${ }^{116}$ P. Sphicas, ${ }^{116, \text { uu }}$ A. Stakia, ${ }^{116}$ J. Steggemann, ${ }^{116}$ M. Tosi, ${ }^{116}$ D. Treille, ${ }^{116}$ A. Tsirou, ${ }^{116}$ V. Veckalns, ${ }^{116, v v}$ W. D. Zeuner, ${ }^{116}$ L. Caminada, ${ }^{117, w w}$ K. Deiters, ${ }^{117}$ W. Erdmann, ${ }^{117}$ R. Horisberger, ${ }^{117}$ Q. Ingram, ${ }^{117}$ H. C. Kaestli, ${ }^{117}$

D. Kotlinski, ${ }^{117}$ U. Langenegger, ${ }^{117}$ T. Rohe, ${ }^{117}$ S. A. Wiederkehr, ${ }^{117}$ M. Backhaus, ${ }^{118}$ L. Bäni, ${ }^{118}$ P. Berger,${ }^{118}$ N. Chernyavskaya, ${ }^{118}$ G. Dissertori, ${ }^{118}$ M. Dittmar, ${ }^{118}$ M. Donegà, ${ }^{118}$ C. Dorfer, ${ }^{118}$ C. Grab, ${ }^{118}$ C. Heidegger, ${ }^{118}$ D. Hits, ${ }^{118}$ J. Hoss, ${ }^{118}$ T. Klijnsma, ${ }^{118}$ W. Lustermann, ${ }^{118}$ R. A. Manzoni, ${ }^{118}$ M. Marionneau, ${ }^{118}$ M. T. Meinhard ${ }^{118}$ F. Micheli, ${ }^{118}$ P. Musella, ${ }^{118}$ F. Nessi-Tedaldi, ${ }^{118}$ J. Pata, ${ }^{118}$ F. Pauss, ${ }^{118}$ G. Perrin, ${ }^{118}$ L. Perrozzi, ${ }^{118}$ S. Pigazzini, ${ }^{118}$ M. Quittnat,${ }^{118}$ D. Ruini, ${ }^{118}$ D. A. Sanz Becerra, ${ }^{118}$ M. Schönenberger, ${ }^{118}$ L. Shchutska, ${ }^{118}$ V. R. Tavolaro, ${ }^{118}$ K. Theofilatos, ${ }^{118}$ M. L. Vesterbacka Olsson, ${ }^{118}$ R. Wallny, ${ }^{118}$ D. H. Zhu, ${ }^{118}$ T. K. Aarrestad, ${ }^{119}$ C. Amsler, ${ }^{19, x x}$ D. Brzhechko, ${ }^{119}$ M. F. Canelli, ${ }^{119}$ A. De Cosa, ${ }^{119}$ R. Del Burgo, ${ }^{119}$ S. Donato, ${ }^{119}$ C. Galloni,${ }^{119}$ T. Hreus, ${ }^{119}$ B. Kilminster, ${ }^{119}$ I. Neutelings, ${ }^{119}$ D. Pinna, ${ }^{119}$ G. Rauco, ${ }^{119}$ P. Robmann, ${ }^{119}$ D. Salerno, ${ }^{119}$ K. Schweiger, ${ }^{119}$ C. Seitz, ${ }^{119}$ Y. Takahashi, ${ }^{119}$ A. Zucchetta, ${ }^{119}$ Y. H. Chang, ${ }^{120}$ K. y. Cheng, ${ }^{120}$ T. H. Doan, ${ }^{120}$ Sh. Jain, ${ }^{120}$ R. Khurana, ${ }^{120}$ C. M. Kuo, ${ }^{120}$ W. Lin, ${ }^{120}$ A. Pozdnyakov, ${ }^{120}$ S. S. Yu, ${ }^{120}$ P. Chang, ${ }^{121}$ Y. Chao, ${ }^{121}$ K. F. Chen, ${ }^{121}$ P. H. Chen, ${ }^{121}$ W.-S. Hou ${ }^{121}$ Arun Kumar, ${ }^{121}$ Y. y. Li, ${ }^{121}$ R.-S. Lu, ${ }^{121}$ E. Paganis, ${ }^{121}$ A. Psallidas, ${ }^{121}$ A. Steen, ${ }^{121}$ J. f. Tsai ${ }^{121}$ B. Asavapibhop, ${ }^{122}$ N. Srimanobhas, ${ }^{122}$ N. Suwonjandee, ${ }^{122}$ A. Bat, ${ }^{123}$ F. Boran, ${ }^{123}$ S. Cerci, ${ }^{123, y y}$ S. Damarseckin, ${ }^{123}$ Z. S. Demiroglu, ${ }^{123}$ F. Dolek, ${ }^{123}$ C. Dozen, ${ }^{123}$ I. Dumanoglu, ${ }^{123}$

S. Girgis, ${ }^{123}$ G. Gokbulut, ${ }^{123}$ Y. Guler, ${ }^{123}$ E. Gurpinar, ${ }^{123}$ I. Hos, ${ }^{123, z z}$ C. Isik, ${ }^{123}$ E. E. Kangal,,${ }^{123, \text { aaa }}$ O. Kara, ${ }^{123}$

A. Kayis Topaksu, ${ }^{123}$ U. Kiminsu, ${ }^{123}$ M. Oglakci, ${ }^{123}$ G. Onengut, ${ }^{123}$ K. Ozdemir, ${ }^{123, \text { bbb }}$ S. Ozturk, ${ }^{123, \text { ccc }}$ D. Sunar Cerci, ${ }^{123, y y}$ B. Tali, ${ }^{123, y y}$ U. G. Tok ${ }^{123}$ S. Turkcapar, ${ }^{123}$ I. S. Zorbakir, ${ }^{123}$ C. Zorbilmez, ${ }^{123}$ B. Isildak, ${ }^{124, d d d}$ G. Karapinar, ${ }^{124, \text { eee }}$ M. Yalvac, ${ }^{124}$ M. Zeyrek, ${ }^{124}$ I. O. Atakisi, ${ }^{125}$ E. Gülmez, ${ }^{125}$ M. Kaya, ${ }^{125, f f}$ O. Kaya, ${ }^{125, g g g}$ S. Tekten, ${ }^{125}$ E. A. Yetkin, ${ }^{125, \text { hhh }}$ M. N. Agaras, ${ }^{126}$ S. Atay, ${ }^{126}$ A. Cakir, ${ }^{126}$ K. Cankocak, ${ }^{126}$ Y. Komurcu, ${ }^{126}$ S. Sen, ${ }^{126, i i i}$ B. Grynyov, ${ }^{127}$ L. Levchuk, ${ }^{128}$

F. Ball, ${ }^{129}$ L. Beck, ${ }^{129}$ J. J. Brooke, ${ }^{129}$ D. Burns, ${ }^{129}$ E. Clement, ${ }^{129}$ D. Cussans, ${ }^{129}$ O. Davignon, ${ }^{129}$ H. Flacher, ${ }^{129}$ J. Goldstein, ${ }^{129}$ G. P. Heath, ${ }^{129}$ H. F. Heath, ${ }^{129}$ L. Kreczko, ${ }^{129}$ D. M. Newbold, ${ }^{129, j j}$ S. Paramesvaran, ${ }^{129}$ B. Penning, ${ }^{129}$ T. Sakuma, ${ }^{129}$ D. Smith, ${ }^{129}$ V. J. Smith, ${ }^{129}$ J. Taylor, ${ }^{129}$ A. Titterton, ${ }^{129}$ K. W. Bell, ${ }^{130}$ A. Belyaev, ${ }^{130, k k k}$ C. Brew, ${ }^{130}$ R. M. Brown, ${ }^{130}$ D. Cieri, ${ }^{130}$ D. J. A. Cockerill, ${ }^{130}$ J. A. Coughlan, ${ }^{130}$ K. Harder ${ }^{130}$ S. Harper,${ }^{130}$ J. Linacre, ${ }^{130}$ E. Olaiya, ${ }^{130}$ D. Petyt, ${ }^{130}$ C. H. Shepherd-Themistocleous, ${ }^{130}$ A. Thea, ${ }^{130}$ I. R. Tomalin, ${ }^{130}$ T. Williams, ${ }^{130}$ W. J. Womersley, ${ }^{130}$ G. Auzinger, ${ }^{131}$ R. Bainbridge, ${ }^{131}$ P. Bloch, ${ }^{131}$ J. Borg ${ }^{131}$ S. Breeze, ${ }^{131}$ O. Buchmuller, ${ }^{131}$ A. Bundock, ${ }^{131}$ S. Casasso, ${ }^{131}$ D. Colling, ${ }^{131}$ L. Corpe ${ }^{131}$ P. Dauncey ${ }^{131}$ G. Davies, ${ }^{131}$ M. Della Negra,${ }^{131}$ R. Di Maria ${ }^{131}$ Y. Haddad,${ }^{131}$ G. Hall, ${ }^{131}$ G. Iles, ${ }^{131}$ T. James, ${ }^{131}$ M. Komm, ${ }^{131}$ C. Laner, ${ }^{131}$ L. Lyons, ${ }^{131}$ A.-M. Magnan, ${ }^{131}$ S. Malik, ${ }^{131}$ A. Martelli, ${ }^{131}$ J. Nash, ${ }^{131,111}$ A. Nikitenko, ${ }^{131, g}$ V. Palladino, ${ }^{131}$ M. Pesaresi, ${ }^{131}$ A. Richards, ${ }^{131}$ A. Rose,${ }^{131}$ E. Scott ${ }^{131}$ C. Seez, ${ }^{131}$ A. Shtipliyski, ${ }^{131}$ G. Singh, ${ }^{131}$ M. Stoye, ${ }^{131}$ T. Strebler, ${ }^{131}$ S. Summers, ${ }^{131}$ A. Tapper,${ }^{131}$ K. Uchida, ${ }^{131}$ T. Virdee,${ }^{131, r}$ N. Wardle, ${ }^{131}$ D. Winterbottom, ${ }^{131}$ J. Wright, ${ }^{131}$ S. C. Zenz, ${ }^{131}$ J. E. Cole, ${ }^{132}$ P. R. Hobson, ${ }^{132}$ A. Khan,${ }^{132}$ P. Kyberd, ${ }^{132}$ C. K. Mackay, ${ }^{132}$ A. Morton, ${ }^{132}$ I. D. Reid, ${ }^{132}$ L. Teodorescu, ${ }^{132}$ S. Zahid, ${ }^{132}$ K. Call, ${ }^{133}$ J. Dittmann, ${ }^{133}$ K. Hatakeyama, ${ }^{133}$ H. Liu, ${ }^{133}$ C. Madrid ${ }^{133}$ B. Mcmaster, ${ }^{133}$ N. Pastika, ${ }^{133}$ C. Smith,${ }^{133}$ R. Bartek, ${ }^{134}$ A. Dominguez, ${ }^{134}$ A. Buccilli, ${ }^{135}$ S. I. Cooper, ${ }^{135}$ C. Henderson, ${ }^{135}$ P. Rumerio, ${ }^{135}$ C. West, ${ }^{135}$ D. Arcaro, ${ }^{136}$ T. Bose, ${ }^{136}$ D. Gastler, ${ }^{136}$ D. Rankin, ${ }^{136}$ C. Richardson, ${ }^{136}$ J. Rohlf, ${ }^{136}$ L. Sulak, ${ }^{136}$ D. Zou, ${ }^{136}$ G. Benelli, ${ }^{137}$ X. Coubez, ${ }^{137}$ D. Cutts, ${ }^{137}$ M. Hadley ${ }_{137}^{137}$ J. Hakala, ${ }^{137}$ U. Heintz, ${ }^{137}$ J. M. Hogan, ${ }^{137, \mathrm{mmm}}$ K. H. M. Kwok, ${ }^{137}$ E. Laird, ${ }^{137}$ G. Landsberg, ${ }^{137}$ J. Lee, ${ }^{137}$ Z. Mao, ${ }^{137}$ M. Narain, ${ }^{137}$ J. Pazzini, ${ }^{137}$ S. Piperov, ${ }^{137}$ S. Sagir, ${ }^{137, n n n}$ R. Syarif, ${ }^{137}$ E. Usai, ${ }^{137}$ D. Yu,${ }^{137}$ R. Band, ${ }^{138}$ C. Brainerd,${ }^{138}$ R. Breedon, ${ }^{138}$ D. Burns, ${ }^{138}$ M. Calderon De La Barca Sanchez, ${ }^{138}$ M. Chertok, ${ }^{138}$ J. Conway, ${ }^{138}$ R. Conway, ${ }^{138}$ P. T. Cox, ${ }^{138}$ R. Erbacher,${ }^{138}$ C. Flores, ${ }^{138}$ G. Funk, ${ }^{138}$ W. Ko, ${ }^{138}$ O. Kukral, ${ }^{138}$ R. Lander, ${ }^{138}$ C. Mclean, ${ }^{138}$ M. Mulhearn, ${ }^{138}$ D. Pellett,,${ }^{138}$ J. Pilot, ${ }^{138}$ S. Shalhout, ${ }^{138}$ M. Shi, ${ }^{138}$ D. Stolp, ${ }^{138}$ D. Taylor, ${ }^{138}$ K. Tos, ${ }^{138}$ M. Tripathi, ${ }^{138}$ Z. Wang, ${ }^{138}$ F. Zhang, ${ }^{138}$ M. Bachtis, ${ }^{139}$ C. Bravo, ${ }^{139}$ R. Cousins, ${ }^{139}$ A. Dasgupta, ${ }^{139}$ A. Florent, ${ }^{139}$ J. Hauser, ${ }^{139}$ M. Ignatenko, ${ }^{139}$ N. Mccoll, ${ }^{139}$ S. Regnard, ${ }^{139}$ D. Saltzberg, ${ }^{139}$ C. Schnaible, ${ }^{139}$ V. Valuev, ${ }^{139}$ E. Bouvier,${ }^{140}$ K. Burt, ${ }^{140}$ R. Clare, ${ }^{140}$ J. W. Gary,${ }^{140}$ S. M. A. Ghiasi Shirazi, ${ }^{140}$ G. Hanson, ${ }^{140}$ G. Karapostoli, ${ }^{140}$ E. Kennedy, ${ }^{140}$ F. Lacroix, ${ }^{140}$ O. R. Long, ${ }^{140}$ M. Olmedo Negrete, ${ }^{140}$ M. I. Paneva, ${ }^{140}$ W. Si, ${ }^{140}$ 
L. Wang, ${ }^{140}$ H. Wei, ${ }^{140}$ S. Wimpenny, ${ }^{140}$ B. R. Yates, ${ }^{140}$ J. G. Branson, ${ }^{141}$ S. Cittolin, ${ }^{141}$ M. Derdzinski, ${ }^{141}$ R. Gerosa, ${ }^{141}$ D. Gilbert, ${ }^{141}$ B. Hashemi, ${ }^{141}$ A. Holzner, ${ }^{141}$ D. Klein,${ }^{141}$ G. Kole ${ }^{141}$ V. Krutelyov,${ }^{141}$ J. Letts, ${ }^{141}$ M. Masciovecchio, ${ }^{141}$ D. Olivito, ${ }^{141}$ S. Padhi, ${ }^{141}$ M. Pieri, ${ }^{141}$ M. Sani, ${ }^{141}$ V. Sharma, ${ }^{141}$ S. Simon,${ }^{141}$ M. Tadel, ${ }^{141}$ A. Vartak,${ }^{141}$

S. Wasserbaech, ${ }^{141,0 o o}$ J. Wood, ${ }^{141}$ F. Würthwein, ${ }^{141}$ A. Yagil, ${ }^{141}$ G. Zevi Della Porta, ${ }^{141}$ N. Amin, ${ }^{142}$ R. Bhandari, ${ }^{142}$ J. Bradmiller-Feld, ${ }^{142}$ C. Campagnari, ${ }^{142}$ M. Citron, ${ }^{142}$ A. Dishaw, ${ }^{142}$ V. Dutta, ${ }^{142}$ M. Franco Sevilla, ${ }^{142}$ L. Gouskos, ${ }^{142}$ R. Heller, ${ }^{142}$ J. Incandela, ${ }^{142}$ A. Ovcharova, ${ }^{142}$ H. Qu, ${ }^{142}$ J. Richman, ${ }^{142}$ D. Stuart, ${ }^{142}$ I. Suarez, ${ }^{142}$ S. Wang, ${ }^{142}$ J. Yoo, ${ }^{142}$ D. Anderson, ${ }^{143}$ A. Bornheim, ${ }^{143}$ J. M. Lawhorn, ${ }^{143}$ H. B. Newman, ${ }^{143}$ T. Q. Nguyen, ${ }^{143}$ M. Spiropulu, ${ }^{143}$ J. R. Vlimant, ${ }^{143}$ R. Wilkinson, ${ }^{143}$ S. Xie, ${ }^{143}$ Z. Zhang, ${ }^{143}$ R. Y. Zhu, ${ }^{143}$ M. B. Andrews, ${ }^{144}$ T. Ferguson, ${ }^{144}$ T. Mudholkar, ${ }^{144}$ M. Paulini, ${ }^{144}$ M. Sun, ${ }^{144}$ I. Vorobiev, ${ }^{144}$ M. Weinberg, ${ }^{144}$ J. P. Cumalat, ${ }^{145}$ W. T. Ford, ${ }^{145}$ F. Jensen, ${ }^{145}$ A. Johnson, ${ }^{145}$ M. Krohn, ${ }^{145}$ S. Leontsinis, ${ }^{145}$ E. MacDonald, ${ }^{145}$ T. Mulholland, ${ }^{145}$ K. Stenson, ${ }^{145}$ K. A. Ulmer, ${ }^{145}$ S. R. Wagner, ${ }^{145}$ J. Alexander, ${ }^{146}$ J. Chaves, ${ }^{146}$ Y. Cheng, ${ }^{146}$ J. Chu, ${ }^{146}$ A. Datta, ${ }^{146}$ K. Mcdermott, ${ }^{146}$ N. Mirman, ${ }^{146}$ J. R. Patterson, ${ }^{146}$ D. Quach, ${ }^{146}$ A. Rinkevicius, ${ }^{146}$ A. Ryd, ${ }^{146}$ L. Skinnari, ${ }^{146}$ L. Soffi, ${ }^{146}$ S. M. Tan, ${ }^{146}$ Z. Tao,${ }^{146}$ J. Thom, ${ }^{146}$ J. Tucker, ${ }^{146}$ P. Wittich, ${ }^{146}$ M. Zientek, ${ }^{146}$ S. Abdullin, ${ }^{147}$ M. Albrow,${ }^{147}$ M. Alyari, ${ }^{147}$ G. Apollinari,${ }^{147}$ A. Apresyan, ${ }^{147}$ A. Apyan, ${ }^{147}$ S. Banerjee, ${ }^{147}$ L. A. T. Bauerdick, ${ }^{147}$ A. Beretvas, ${ }^{147}$ J. Berryhill, ${ }^{147}$ P. C. Bhat, ${ }^{147}$ G. Bolla, ${ }^{147, a}$ K. Burkett, ${ }^{147}$ J. N. Butler, ${ }^{147}$ A. Canepa, ${ }^{147}$ G. B. Cerati, ${ }^{147}$ H. W. K. Cheung, ${ }^{147}$ F. Chlebana, ${ }^{147}$ M. Cremonesi, ${ }^{147}$ J. Duarte, ${ }^{147}$ V. D. Elvira, ${ }^{147}$ J. Freeman, ${ }^{147}$ Z. Gecse, ${ }^{147}$ E. Gottschalk, ${ }^{147}$ L. Gray, ${ }^{147}$ D. Green, ${ }^{147}$ S. Grünendahl, ${ }^{147}$ O. Gutsche, ${ }^{147}$ J. Hanlon, ${ }^{147}$ R. M. Harris, ${ }^{147}$ S. Hasegawa, ${ }^{147}$ J. Hirschauer, ${ }^{147}$ Z. Hu, ${ }^{147}$ B. Jayatilaka, ${ }^{147}$ S. Jindariani, ${ }^{147}$ M. Johnson, ${ }^{147}$ U. Joshi, ${ }^{147}$ B. Klima, ${ }^{147}$ M. J. Kortelainen, ${ }^{147}$ B. Kreis, ${ }^{147}$ S. Lammel, ${ }^{147}$ D. Lincoln, ${ }^{147}$ R. Lipton, ${ }^{147}$ M. Liu, ${ }^{147}$ T. Liu, ${ }^{147}$ J. Lykken, ${ }^{147}$ K. Maeshima, ${ }^{147}$ J. M. Marraffino, ${ }^{147}$ D. Mason, ${ }^{147}$ P. McBride, ${ }^{147}$ P. Merkel,,${ }^{147}$ S. Mrenna, ${ }^{147}$ S. Nahn, ${ }^{147}$ V. O’Dell, ${ }^{147}$ K. Pedro, ${ }^{147}$ C. Pena, ${ }^{147}$ O. Prokofyev, ${ }^{147}$ G. Rakness, ${ }^{147}$ L. Ristori, ${ }^{147}$ A. Savoy-Navarro, ${ }^{147, p p p}$ B. Schneider, ${ }^{147}$ E. Sexton-Kennedy, ${ }^{147}$ A. Soha, ${ }^{147}$ W. J. Spalding,${ }^{147}$ L. Spiegel ${ }^{147}$ S. Stoynev, ${ }^{147}$ J. Strait,${ }^{147}$ N. Strobbe,${ }^{147}$ L. Taylor, ${ }^{147}$ S. Tkaczyk, ${ }^{147}$ N. V. Tran, ${ }^{147}$ L. Uplegger, ${ }^{147}$ E. W. Vaandering, ${ }^{147}$ C. Vernieri, ${ }^{147}$ M. Verzocchi, ${ }^{147}$ R. Vidal, ${ }^{147}$ M. Wang, ${ }^{147}$ H. A. Weber, ${ }^{147}$ A. Whitbeck, ${ }^{147}$ D. Acosta, ${ }^{148}$ P. Avery, ${ }^{148}$ P. Bortignon, ${ }^{148}$ D. Bourilkov, ${ }^{148}$ A. Brinkerhoff, ${ }^{148}$ L. Cadamuro, ${ }^{148}$ A. Carnes, ${ }^{148}$ M. Carver, ${ }^{148}$ D. Curry, ${ }^{148}$ R. D. Field, ${ }^{148}$ S. V. Gleyzer, ${ }^{148}$ B. M. Joshi, ${ }^{148}$ J. Konigsberg, ${ }^{148}$ A. Korytov, ${ }^{148}$ P. Ma, ${ }^{148}$ K. Matchev,${ }^{148}$ H. Mei, ${ }^{148}$ G. Mitselmakher, ${ }^{148}$ K. Shi, ${ }^{148}$ D. Sperka, ${ }^{148}$ J. Wang, ${ }^{148}$ S. Wang, ${ }^{148}$ Y. R. Joshi, ${ }^{149}$ S. Linn, ${ }^{149}$ A. Ackert, ${ }^{150}$ T. Adams, ${ }^{150}$ A. Askew, ${ }^{150}$ S. Hagopian, ${ }^{150}$ V. Hagopian, ${ }^{150}$ K. F. Johnson, ${ }^{150}$

T. Kolberg, ${ }^{150}$ G. Martinez, ${ }^{150}$ T. Perry, ${ }^{150}$ H. Prosper, ${ }^{150}$ A. Saha, ${ }^{150}$ A. Santra,${ }^{150}$ V. Sharma,${ }^{150}$ R. Yohay, ${ }^{150}$

M. M. Baarmand, ${ }^{151}$ V. Bhopatkar, ${ }^{151}$ S. Colafranceschi, ${ }^{151}$ M. Hohlmann, ${ }^{151}$ D. Noonan, ${ }^{151}$ M. Rahmani ${ }^{151}$ T. Roy, ${ }^{151}$ F. Yumiceva, ${ }^{151}$ M. R. Adams, ${ }^{152}$ L. Apanasevich, ${ }^{152}$ D. Berry, ${ }^{152}$ R. R. Betts,${ }^{152}$ R. Cavanaugh, ${ }^{152}$ X. Chen, ${ }^{152}$ S. Dittmer, ${ }^{152}$ O. Evdokimov, ${ }^{152}$ C. E. Gerber, ${ }^{152}$ D. A. Hangal, ${ }^{152}$ D. J. Hofman, ${ }^{152}$ K. Jung, ${ }^{152}$ J. Kamin, ${ }^{152}$ C. Mills, ${ }^{152}$ I. D. Sandoval Gonzalez, ${ }^{152}$ M. B. Tonjes ${ }^{152}$ N. Varelas, ${ }^{152}$ H. Wang, ${ }^{152}$ X. Wang, ${ }^{152}$ Z. Wu, ${ }^{152}$ J. Zhang, ${ }^{152}$ M. Alhusseini, ${ }_{153}$ B. Bilki, ${ }^{153, q q q}$ W. Clarida, ${ }^{153}$ K. Dilsiz, ${ }^{153, \text { rrr }}$ S. Durgut, ${ }^{153}$ R. P. Gandrajula, ${ }^{153}$ M. Haytmyradov, ${ }^{153}$ V. Khristenko, ${ }^{153}$ J.-P. Merlo, ${ }^{153}$ A. Mestvirishvili, ${ }^{153}$ A. Moeller, ${ }^{153}$ J. Nachtman, ${ }^{153}$ H. Ogul, ${ }^{153, \text { sss }}$ Y. Onel,,${ }^{153}$ F. Ozok, ${ }^{153, \text { ttt }}$ A. Penzo, ${ }^{153}$ C. Snyder, ${ }^{153}$ E. Tiras, ${ }^{153}$ J. Wetzel, ${ }^{153}$ B. Blumenfeld, ${ }^{154}$ A. Cocoros, ${ }^{154}$ N. Eminizer, ${ }^{154}$ D. Fehling, ${ }^{154}$ L. Feng, ${ }^{154}$ A. V. Gritsan, ${ }^{154}$ W. T. Hung, ${ }^{154}$ P. Maksimovic, ${ }^{154}$ J. Roskes, ${ }^{154}$ U. Sarica, ${ }^{154}$ M. Swartz, ${ }^{154}$ M. Xiao, ${ }^{154}$ C. You, ${ }^{154}$ A. Al-bataineh, ${ }^{155}$ P. Baringer, ${ }^{155}$ A. Bean, ${ }^{155}$ S. Boren, ${ }^{155}$ J. Bowen, ${ }^{155}$ A. Bylinkin, ${ }^{155}$ J. Castle, ${ }^{155}$ S. Khalil, ${ }^{155}$ A. Kropivnitskaya ${ }^{155}$ D. Majumder, ${ }^{155}$ W. Mcbrayer, ${ }^{155}$ M. Murray ${ }^{155}$ C. Rogan, ${ }^{155}$ S. Sanders, ${ }^{155}$ E. Schmitz, ${ }^{155}$ J. D. Tapia Takaki, ${ }^{155}$ Q. Wang, ${ }_{155}^{15}$ A. Ivanov, ${ }^{156}$ K. Kaadze, ${ }^{156}$ D. Kim, ${ }^{156}$ Y. Maravin, ${ }^{156}$ D. R. Mendis, ${ }^{156}$ T. Mitchell, ${ }^{156}$ A. Modak, ${ }^{156}$ A. Mohammadi, ${ }^{156}$ L. K. Saini, ${ }^{156}$ N. Skhirtladze, ${ }^{156}$ F. Rebassoo, ${ }^{157}$ D. Wright, ${ }^{157}$ A. Baden, ${ }^{158}$ O. Baron, ${ }^{158}$ A. Belloni, ${ }^{158}$ S. C. Eno, ${ }^{158}$ Y. Feng, ${ }_{158}$ C. Ferraioli, ${ }^{158}$ N. J. Hadley,${ }^{158}$ S. Jabeen, ${ }^{158}$ G. Y. Jeng, ${ }_{158}^{158}$ R. G. Kellogg ${ }^{158}$

J. Kunkle, ${ }^{158}$ A. C. Mignerey, ${ }^{158}$ F. Ricci-Tam, ${ }^{158}$ Y. H. Shin, ${ }^{158}$ A. Skuja, ${ }^{158}$ S. C. Tonwar, ${ }^{158}$ K. Wong, ${ }^{158}$ D. Abercrombie, ${ }^{159}$ B. Allen, ${ }^{159}$ V. Azzolini, ${ }^{159}$ A. Baty, ${ }^{159}$ G. Bauer, ${ }^{159}$ R. Bi, ${ }^{159}$ S. Brandt,${ }^{159}$ W. Busza, ${ }^{159}$ I. A. Cali, ${ }^{159}$ M. D’ Alfonso, ${ }^{159}$ Z. Demiragli ${ }^{159}$ G. Gomez Ceballos, ${ }^{159}$ M. Goncharov, ${ }^{159}$ P. Harris, ${ }^{159}$ D. Hsu, ${ }^{159}$ M. Hu, ${ }^{159}$ Y. Iiyama, ${ }^{159}$ G. M. Innocenti, ${ }^{159}$ M. Klute, ${ }^{159}$ D. Kovalskyi, ${ }^{159}$ Y.-J. Lee, ${ }^{159}$ P. D. Luckey, ${ }^{159}$ B. Maier, ${ }^{159}$ A. C. Marini, ${ }^{159}$ C. Mcginn, ${ }^{159}$ C. Mironov, ${ }^{159}$ S. Narayanan, ${ }^{159}$ X. Niu, ${ }^{159}$ C. Paus, ${ }^{159}$ C. Roland, ${ }^{159}$ G. Roland, ${ }^{159}$ G. S. F. Stephans, ${ }^{159}$ K. Sumorok, ${ }^{159}$

K. Tatar, ${ }^{159}$ D. Velicanu, ${ }^{159}$ J. Wang, ${ }^{159}$ T. W. Wang, ${ }^{159}$ B. Wyslouch, ${ }^{159}$ S. Zhaozhong, ${ }^{159}$ A. C. Benvenuti, ${ }^{160}$ R. M. Chatterjee, ${ }^{160}$ A. Evans, ${ }^{160}$ P. Hansen, ${ }^{160}$ S. Kalafut, ${ }^{160}$ Y. Kubota, ${ }^{160}$ Z. Lesko, ${ }^{160}$ J. Mans, ${ }^{160}$ S. Nourbakhsh, ${ }^{160}$ N. Ruckstuhl ${ }^{160}$ R. Rusack, ${ }^{160}$ J. Turkewitz ${ }^{160}$ M. A. Wadud, ${ }^{160}$ J. G. Acosta, ${ }^{161}$ S. Oliveros, ${ }^{161}$ E. Avdeeva, ${ }^{162}$ K. Bloom, ${ }^{162}$ D. R. Claes, ${ }^{162}$ C. Fangmeier, ${ }^{162}$ F. Golf, ${ }^{162}$ R. Gonzalez Suarez, ${ }^{162}$ R. Kamalieddin, ${ }^{162}$ I. Kravchenko, ${ }^{162}$ J. Monroy, ${ }^{162}$ 
J. E. Siado, ${ }^{162}$ G. R. Snow, ${ }^{162}$ B. Stieger, ${ }^{162}$ A. Godshalk, ${ }^{163}$ C. Harrington, ${ }^{163}$ I. Iashvili, ${ }^{163}$ A. Kharchilava, ${ }^{163}$ D. Nguyen, ${ }^{163}$ A. Parker, ${ }^{163}$ S. Rappoccio, ${ }^{163}$ B. Roozbahani, ${ }^{163}$ G. Alverson, ${ }^{164}$ E. Barberis, ${ }^{164}$ C. Freer, ${ }^{164}$ A. Hortiangtham, ${ }^{164}$ D. M. Morse, ${ }^{164}$ T. Orimoto, ${ }^{164}$ R. Teixeira De Lima, ${ }^{164}$ T. Wamorkar, ${ }^{164}$ B. Wang, ${ }^{164}$ A. Wisecarver, ${ }^{164}$ D. Wood, ${ }^{164}$ S. Bhattacharya, ${ }^{165}$ O. Charaf,${ }^{165}$ K. A. Hahn, ${ }^{165}$ N. Mucia, ${ }^{165}$ N. Odell, ${ }^{165}$ M. H. Schmitt, ${ }^{165}$ K. Sung, ${ }^{165}$ M. Trovato, ${ }^{165}$ M. Velasco, ${ }^{165}$ R. Bucci, ${ }^{166}$ N. Dev,${ }^{166}$ M. Hildreth, ${ }^{166}$ K. Hurtado Anampa,${ }^{166}$ C. Jessop, ${ }^{166}$ D. J. Karmgard, ${ }^{166}$ N. Kellams, ${ }^{166}$ K. Lannon, ${ }^{166}$ W. Li, ${ }^{166}$ N. Loukas, ${ }^{166}$ N. Marinelli, ${ }^{166}$ F. Meng, ${ }^{166}$ C. Mueller, ${ }^{166}$ Y. Musienko, ${ }^{166, j j}$ M. Planer, ${ }^{166}$ A. Reinsvold, ${ }^{166}$ R. Ruchti, ${ }^{166}$ P. Siddireddy, ${ }^{166}$ G. Smith, ${ }^{166}$ S. Taroni, ${ }^{166}$ M. Wayne, ${ }^{166}$ A. Wightman, ${ }^{166}$ M. Wolf, ${ }^{166}$ A. Woodard, ${ }^{166}$ J. Alimena, ${ }^{167}$ L. Antonelli, ${ }^{167}$ B. Bylsma, ${ }^{167}$ L. S. Durkin, ${ }^{167}$ S. Flowers, ${ }^{167}$ B. Francis, ${ }^{167}$ A. Hart, ${ }^{167}$ C. Hill, ${ }^{167}$ W. Ji, ${ }^{167}$ T. Y. Ling, ${ }^{167}$ W. Luo, ${ }^{167}$ B. L. Winer, ${ }^{167}$ H. W. Wulsin, ${ }^{167}$ S. Cooperstein, ${ }^{168}$ P. Elmer, ${ }^{168}$ J. Hardenbrook, ${ }^{168}$ P. Hebda, ${ }^{168}$ S. Higginbotham, ${ }^{168}$ A. Kalogeropoulos, ${ }^{168}$ D. Lange, ${ }^{168}$ M. T. Lucchini, ${ }^{168}$ J. Luo, ${ }^{168}$ D. Marlow, ${ }^{168}$ K. Mei, ${ }^{168}$ I. Ojalvo, ${ }^{168}$ J. Olsen, ${ }^{168}$ C. Palmer, ${ }^{168}$ P. Piroué, ${ }^{168}$ J. Salfeld-Nebgen, ${ }^{168}$ D. Stickland, ${ }^{168}$ C. Tully, ${ }^{168}$ S. Malik, ${ }^{169}$ S. Norberg, ${ }^{169}$ A. Barker ${ }^{170}$ V. E. Barnes, ${ }^{170}$ S. Das, ${ }^{170}$ L. Gutay, ${ }^{170}$ M. Jones, ${ }^{170}$ A. W. Jung, ${ }^{170}$ A. Khatiwada, ${ }^{170}$ B. Mahakud, ${ }^{170}$ D. H. Miller, ${ }^{170}$ N. Neumeister, ${ }^{170}$ C. C. Peng, ${ }^{170}$ H. Qiu, ${ }^{170}$ J. F. Schulte, ${ }^{170}$ J. Sun, ${ }^{170}$ F. Wang, ${ }^{170}$ R. Xiao, ${ }^{170}$ W. Xie, ${ }^{170}$ T. Cheng, ${ }^{171}$ J. Dolen, ${ }^{171}$ N. Parashar, ${ }^{171}$ Z. Chen, ${ }^{172}$ K. M. Ecklund, ${ }^{172}$ S. Freed, ${ }^{172}$ F. J. M. Geurts, ${ }^{172}$ M. Kilpatrick, ${ }^{172}$ W. Li,,${ }^{172}$ B. Michlin, ${ }^{172}$ B. P. Padley, ${ }^{172}$ J. Roberts, ${ }^{172}$ J. Rorie, ${ }^{172}$ W. Shi, ${ }^{172}$ Z. Tu, ${ }^{172}$ J. Zabel ${ }^{172}$ A. Zhang, ${ }^{172}$ A. Bodek, ${ }^{173}$ P. de Barbaro, ${ }^{173}$ R. Demina, ${ }^{173}$ Y. t. Duh, ${ }^{173}$ J. L. Dulemba, ${ }^{173}$ C. Fallon, ${ }^{173}$ T. Ferbel, ${ }^{173}$ M. Galanti, ${ }^{173}$ A. Garcia-Bellido, ${ }^{173}$ J. Han, ${ }^{173}$ O. Hindrichs, ${ }^{173}$ A. Khukhunaishvili, ${ }^{173}$ K. H. Lo,${ }^{173}$ P. Tan, ${ }^{173}$ R. Taus, ${ }^{173}$ M. Verzetti, ${ }^{173}$ A. Agapitos, ${ }^{174}$ J. P. Chou, ${ }^{174}$ Y. Gershtein, ${ }^{174}$ T. A. Gómez Espinosa, ${ }^{174}$ E. Halkiadakis, ${ }^{174}$ M. Heindl, ${ }^{174}$ E. Hughes, ${ }^{174}$ S. Kaplan, ${ }^{174}$ R. Kunnawalkam Elayavalli, ${ }^{174}$ S. Kyriacou, ${ }^{174}$ A. Lath, ${ }^{174}$ R. Montalvo, ${ }^{174}$ K. Nash, ${ }^{174}$ M. Osherson, ${ }^{174}$ H. Saka, ${ }^{174}$ S. Salur, ${ }^{174}$ S. Schnetzer, ${ }^{174}$ D. Sheffield, ${ }^{174}$ S. Somalwar, ${ }^{174}$ R. Stone, ${ }^{174}$ S. Thomas, ${ }^{174}$ P. Thomassen, ${ }^{174}$ M. Walker, ${ }^{174}$ A. G. Delannoy ${ }^{175}$ J. Heideman, ${ }^{175}$ G. Riley, ${ }^{175}$ K. Rose, ${ }^{175}$ S. Spanier, ${ }^{175}$ K. Thapa ${ }^{175}$ O. Bouhali, ${ }^{176, \text { uuu }}$ A. Celik, ${ }^{176}$ M. Dalchenko, ${ }^{176}$ M. De Mattia, ${ }^{176}$ A. Delgado, ${ }^{176}$ S. Dildick, ${ }^{176}$ R. Eusebi, ${ }^{176}$ J. Gilmore, ${ }^{176}$ T. Huang, ${ }^{176}$ T. Kamon, ${ }^{176, v v v}$ S. Luo, ${ }^{176}$ R. Mueller, ${ }^{176}$ Y. Pakhotin, ${ }^{176}$ R. Patel,,${ }^{176}$ A. Perloff, ${ }^{176}$ L. Perniè, ${ }^{176}$ D. Rathjens, ${ }^{176}$ A. Safonov, ${ }^{176}$ A. Tatarinov, ${ }^{176}$ N. Akchurin, ${ }^{177}$ J. Damgov, ${ }^{177}$ F. De Guio, ${ }^{177}$ P. R. Dudero, ${ }^{177}$ S. Kunori, ${ }^{177}$ K. Lamichhane, ${ }^{177}$ S. W. Lee, ${ }^{177}$ T. Mengke, ${ }^{177}$ S. Muthumuni, ${ }^{177}$ T. Peltola, ${ }^{177}$ S. Undleeb,${ }^{177}$ I. Volobouev, ${ }^{177}$ Z. Wang, ${ }^{177}$ S. Greene, ${ }^{178}$ A. Gurrola, ${ }^{178}$ R. Janjam, ${ }^{178}$ W. Johns, ${ }^{178}$ C. Maguire, ${ }^{178}$ A. Melo, ${ }^{178} \mathrm{H}$. Ni, ${ }^{178}$ K. Padeken, ${ }^{178}$

J. D. Ruiz Alvarez, ${ }^{178}$ P. Sheldon, ${ }^{178}$ S. Tuo, ${ }^{178}$ J. Velkovska, ${ }^{178}$ M. Verweij, ${ }^{178}$ Q. Xu, ${ }^{178}$ M. W. Arenton, ${ }^{179}$ P. Barria, ${ }^{179}$ B. Cox,${ }^{179}$ R. Hirosky, ${ }^{179}$ M. Joyce, ${ }^{179}$ A. Ledovskoy, ${ }^{179}$ H. Li, ${ }^{179}$ C. Neu, ${ }^{179}$ T. Sinthuprasith,,${ }^{179}$ Y. Wang, ${ }^{179}$ E. Wolfe, ${ }^{179}$ F. Xia, ${ }^{179}$ R. Harr, ${ }^{180}$ P. E. Karchin, ${ }^{180}$ N. Poudyal, ${ }^{180}$ J. Sturdy, ${ }^{180}$ P. Thapa,${ }^{180}$ S. Zaleski, ${ }^{180}$ M. Brodski, ${ }^{181}$ J. Buchanan, ${ }^{181}$ C. Caillol, ${ }^{181}$ D. Carlsmith ${ }^{181}$ S. Dasu, ${ }^{181}$ L. Dodd,${ }^{181}$ S. Duric, ${ }^{181}$ B. Gomber ${ }^{181}$ M. Grothe, ${ }^{181}$ M. Herndon, ${ }^{181}$ A. Hervé, ${ }^{181}$ U. Hussain, ${ }^{181}$ P. Klabbers, ${ }^{181}$ A. Lanaro, ${ }^{181}$ A. Levine, ${ }^{181}$ K. Long, ${ }^{181}$ R. Loveless, ${ }^{181}$ T. Ruggles, ${ }^{181}$ A. Savin, ${ }^{181}$ N. Smith, ${ }^{181}$ W. H. Smith, ${ }^{181}$ and N. Woods ${ }^{181}$

\section{(CMS Collaboration)}

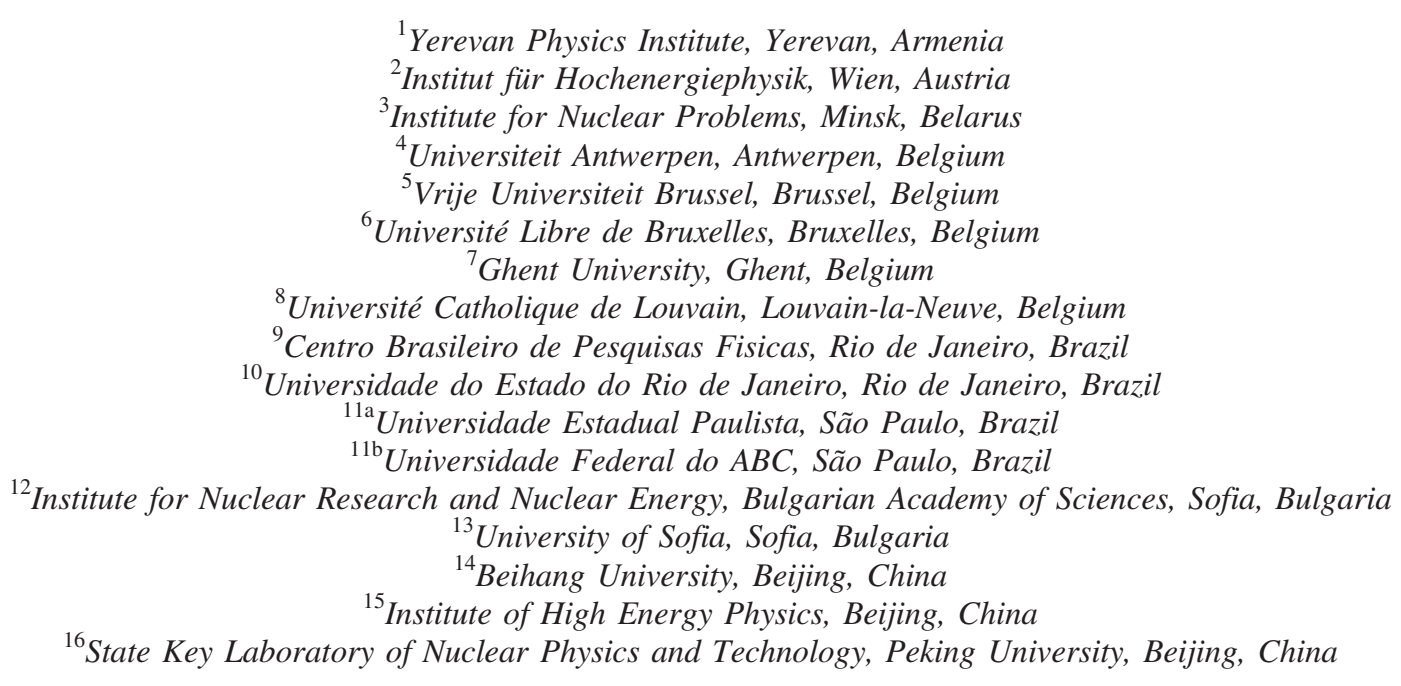




\footnotetext{
${ }^{17}$ Tsinghua University, Beijing, China

${ }^{18}$ Universidad de Los Andes, Bogota, Colombia
}

${ }^{19}$ University of Split, Faculty of Electrical Engineering, Mechanical Engineering and Naval Architecture, Split, Croatia

${ }^{20}$ University of Split, Faculty of Science, Split, Croatia

${ }^{21}$ Institute Rudjer Boskovic, Zagreb, Croatia

${ }^{22}$ University of Cyprus, Nicosia, Cyprus

${ }^{23}$ Charles University, Prague, Czech Republic

${ }^{24}$ Escuela Politecnica Nacional, Quito, Ecuador

${ }^{25}$ Universidad San Francisco de Quito, Quito, Ecuador

${ }^{26}$ Academy of Scientific Research and Technology of the Arab Republic of Egypt,

Egyptian Network of High Energy Physics, Cairo, Egypt

${ }^{27}$ National Institute of Chemical Physics and Biophysics, Tallinn, Estonia

${ }^{28}$ Department of Physics, University of Helsinki, Helsinki, Finland

${ }^{29}$ Helsinki Institute of Physics, Helsinki, Finland

${ }^{30}$ Lappeenranta University of Technology, Lappeenranta, Finland

${ }^{31}$ IRFU, CEA, Université Paris-Saclay, Gif-sur-Yvette, France

${ }^{32}$ Laboratoire Leprince-Ringuet, Ecole polytechnique, CNRS/IN2P3, Université Paris-Saclay, Palaiseau, France

${ }^{33}$ Université de Strasbourg, CNRS, IPHC UMR 7178, Strasbourg, France

${ }^{34}$ Centre de Calcul de l'Institut National de Physique Nucleaire et de Physique des Particules, CNRS/IN2P3, Villeurbanne, France

${ }^{35}$ Université de Lyon, Université Claude Bernard Lyon 1, CNRS-IN2P3, Institut de Physique Nucléaire de Lyon, Villeurbanne, France

${ }^{36}$ Georgian Technical University, Tbilisi, Georgia

${ }^{37}$ Tbilisi State University, Tbilisi, Georgia

${ }^{38}$ RWTH Aachen University, I. Physikalisches Institut, Aachen, Germany

${ }^{39}$ RWTH Aachen University, III. Physikalisches Institut A, Aachen, Germany

${ }^{40}$ RWTH Aachen University, III. Physikalisches Institut B, Aachen, Germany

${ }^{41}$ Deutsches Elektronen-Synchrotron, Hamburg, Germany

${ }^{42}$ University of Hamburg, Hamburg, Germany

${ }^{43}$ Karlsruher Institut fuer Technology

${ }^{44}$ Institute of Nuclear and Particle Physics (INPP), NCSR Demokritos, Aghia Paraskevi, Greece

${ }^{45}$ National and Kapodistrian University of Athens, Athens, Greece

${ }^{46}$ National Technical University of Athens, Athens, Greece

${ }^{47}$ University of Ioánnina, Ioánnina, Greece

${ }^{48}$ MTA-ELTE Lendület CMS Particle and Nuclear Physics Group, Eötvös Loránd University, Budapest, Hungary

${ }^{49}$ Wigner Research Centre for Physics, Budapest, Hungary

${ }^{50}$ Institute of Nuclear Research ATOMKI, Debrecen, Hungary

${ }^{51}$ Institute of Physics, University of Debrecen, Debrecen, Hungary

${ }^{52}$ Indian Institute of Science (IISc), Bangalore, India

${ }^{53}$ National Institute of Science Education and Research, HBNI, Bhubaneswar, India

${ }^{54}$ Panjab University, Chandigarh, India

${ }^{55}$ University of Delhi, Delhi, India

${ }^{56}$ Saha Institute of Nuclear Physics, HBNI, Kolkata,India

${ }^{57}$ Indian Institute of Technology Madras, Madras, India

${ }^{58}$ Bhabha Atomic Research Centre, Mumbai, India

${ }^{59}$ Tata Institute of Fundamental Research-A, Mumbai, India

${ }^{60}$ Tata Institute of Fundamental Research-B, Mumbai, India

${ }^{61}$ Indian Institute of Science Education and Research (IISER), Pune, India

${ }^{62}$ Institute for Research in Fundamental Sciences (IPM), Tehran, Iran

${ }^{63}$ University College Dublin, Dublin, Ireland

${ }^{64 a}$ INFN Sezione di Bari, Bari, Italy

${ }^{64 \mathrm{~b}}$ Università di Bari, Bari, Italy

${ }^{64 \mathrm{c}}$ Politecnico di Bari, Bari, Italy

${ }^{65 \mathrm{a}}$ INFN Sezione di Bologna, Bologna, Italy

${ }^{65 \mathrm{~b}}$ Università di Bologna, Bologna, Italy

${ }^{66 a}$ INFN Sezione di Catania, Catania, Italy

${ }^{66 \mathrm{~b}}$ Università di Catania, Catania, Italy 


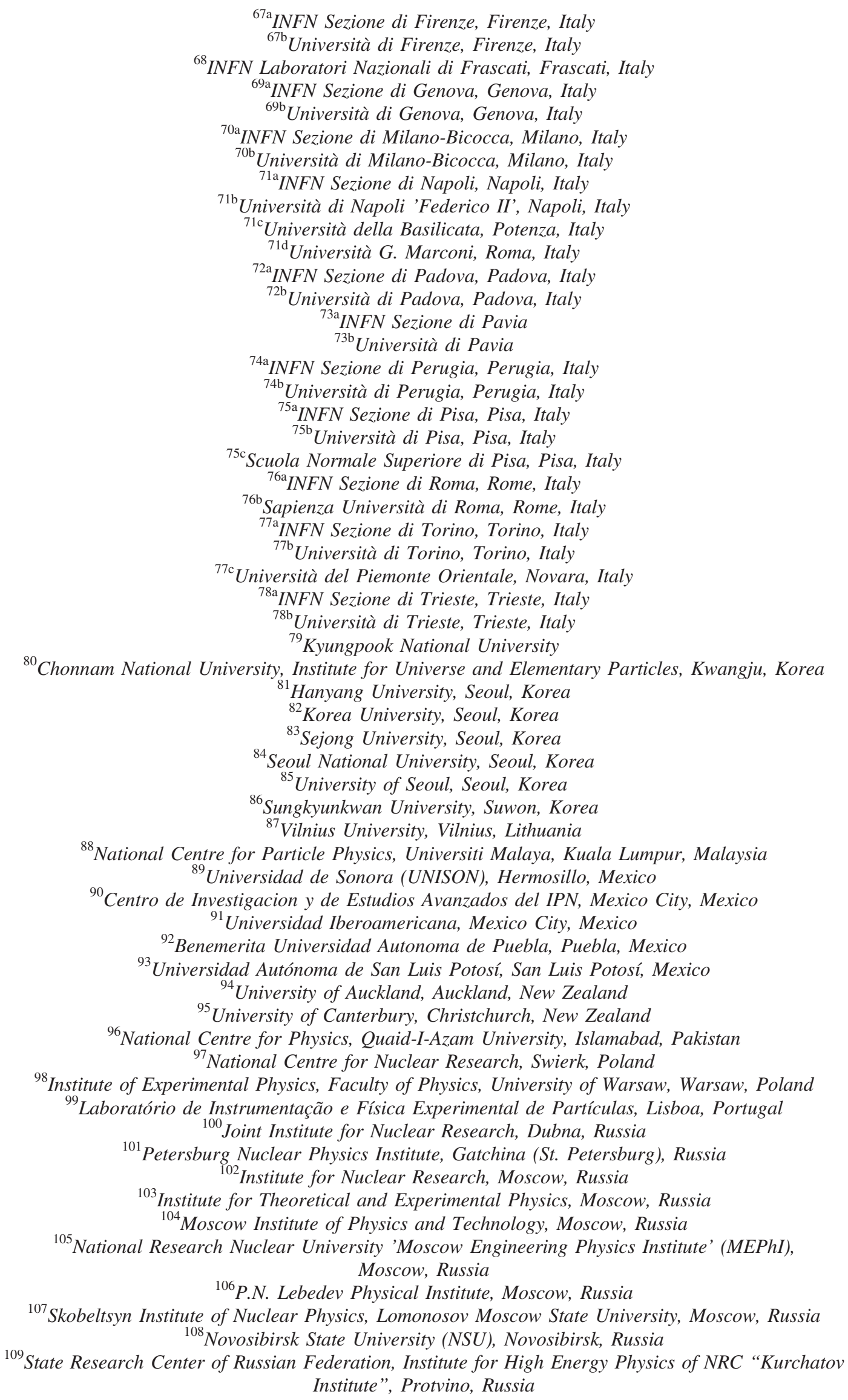


${ }^{110}$ National Research Tomsk Polytechnic University, Tomsk, Russia

${ }^{111}$ University of Belgrade, Faculty of Physics and Vinca Institute of Nuclear Sciences, Belgrade, Serbia

${ }^{112}$ Centro de Investigaciones Energéticas Medioambientales y Tecnológicas (CIEMAT), Madrid, Spain

${ }^{113}$ Universidad Autónoma de Madrid, Madrid, Spain

${ }^{114}$ Universidad de Oviedo, Oviedo, Spain

${ }^{115}$ Instituto de Física de Cantabria (IFCA), CSIC-Universidad de Cantabria, Santander, Spain

${ }^{116}$ CERN, European Organization for Nuclear Research, Geneva, Switzerland

${ }^{117}$ Paul Scherrer Institut, Villigen, Switzerland

${ }^{118}$ ETH Zurich-Institute for Particle Physics and Astrophysics (IPA), Zurich, Switzerland

${ }^{119}$ Universität Zürich, Zurich, Switzerland

${ }^{120}$ National Central University, Chung-Li, Taiwan

${ }^{121}$ National Taiwan University (NTU), Taipei, Taiwan

${ }^{122}$ Chulalongkorn University, Faculty of Science, Department of Physics, Bangkok, Thailand

${ }^{123}$ Çukurova University, Physics Department, Science and Art Faculty, Adana, Turkey

${ }^{124}$ Middle East Technical University, Physics Department, Ankara, Turkey

${ }^{125}$ Bogazici University, Istanbul, Turkey

${ }^{126}$ Istanbul Technical University, Istanbul, Turkey

${ }^{127}$ Institute for Scintillation Materials of National Academy of Science of Ukraine, Kharkov, Ukraine

${ }^{128}$ National Scientific Center, Kharkov Institute of Physics and Technology, Kharkov, Ukraine

${ }^{129}$ University of Bristol, Bristol, United Kingdom

${ }^{130}$ Rutherford Appleton Laboratory, Didcot, United Kingdom

${ }^{131}$ Imperial College, London, United Kingdom

${ }^{132}$ Brunel University, Uxbridge, United Kingdom

${ }^{133}$ Baylor University, Waco, Texas, USA

${ }^{134}$ Catholic University of America, Washington, DC, USA

${ }^{135}$ The University of Alabama, Tuscaloosa, Alabama, USA

${ }^{136}$ Boston University, Boston, Massachusetts, USA

${ }^{137}$ Brown University, Providence, Rhode Island, USA

${ }^{138}$ University of California, Davis, Davis, California, USA

${ }^{139}$ University of California, Los Angeles, California, USA

${ }^{140}$ University of California, Riverside, Riverside, California, USA

${ }^{141}$ University of California, San Diego, La Jolla, California, USA

${ }^{142}$ University of California, Santa Barbara-Department of Physics, Santa Barbara, California, USA

${ }^{143}$ California Institute of Technology, Pasadena, California, USA

${ }^{144}$ Carnegie Mellon University, Pittsburgh, Pennsylvania, USA

${ }^{145}$ University of Colorado Boulder, Boulder, Colorado, USA

${ }^{146}$ Cornell University, Ithaca, New York, USA

${ }^{147}$ Fermi National Accelerator Laboratory, Batavia, Illinois, USA

${ }^{148}$ University of Florida, Gainesville, Florida, USA

${ }^{149}$ Florida International University, Miami, Florida, USA

${ }^{150}$ Florida State University, Tallahassee, Florida, USA

${ }^{151}$ Florida Institute of Technology, Melbourne, Florida, USA

${ }^{152}$ University of Illinois at Chicago (UIC), Chicago, Illinois, USA

${ }^{153}$ The University of Iowa, Iowa City, Iowa, USA

${ }^{154}$ Johns Hopkins University, Baltimore, Maryland, USA

${ }^{155}$ The University of Kansas, Lawrence, Kansas, USA

${ }^{156}$ Kansas State University, Manhattan, Kansas, USA

${ }^{157}$ Lawrence Livermore National Laboratory, Livermore, California, USA

${ }^{158}$ University of Maryland, College Park, Maryland, USA

${ }^{159}$ Massachusetts Institute of Technology, Cambridge, Massachusetts, USA

${ }^{160}$ University of Minnesota, Minneapolis, Minnesota, USA

${ }^{161}$ University of Mississippi, Oxford, Mississippi, USA

${ }^{162}$ University of Nebraska-Lincoln, Lincoln, Nebraska, USA

${ }^{163}$ State University of New York at Buffalo, Buffalo, New York, USA

${ }^{164}$ Northeastern University, Boston, Massachusetts, USA

${ }^{165}$ Northwestern University, Evanston, Illinois, USA

${ }^{166}$ University of Notre Dame, Notre Dame, Indiana, USA

${ }^{167}$ The Ohio State University, Columbus, Ohio, USA

${ }^{168}$ Princeton University, Princeton, New Jersey, USA

${ }^{169}$ University of Puerto Rico, Mayaguez, Puerto Rico 


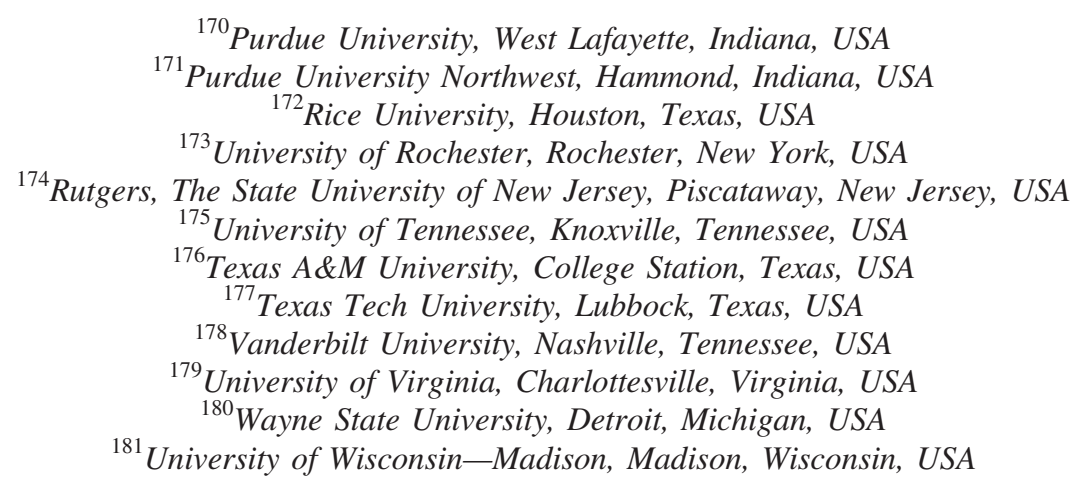

${ }^{\mathrm{a}}$ Deceased.

${ }^{\mathrm{b}}$ Also at Vienna University of Technology, Vienna, Austria.

${ }^{\mathrm{c}}$ Also at IRFU, CEA, Université Paris-Saclay, Gif-sur-Yvette, France.

${ }^{\mathrm{d}}$ Also at Universidade Estadual de Campinas, Campinas, Brazil.

${ }^{\mathrm{e}}$ Also at Federal University of Rio Grande do Sul, Porto Alegre, Brazil.

${ }^{\mathrm{f}}$ Also at Université Libre de Bruxelles, Bruxelles, Belgium.

${ }^{\mathrm{g}}$ Also at Institute for Theoretical and Experimental Physics, Moscow, Russia.

${ }^{\mathrm{h}}$ Also at Joint Institute for Nuclear Research, Dubna, Russia.

${ }^{\mathrm{i}}$ Also at Helwan University, Cairo, Egypt.

${ }^{\mathrm{j}}$ Also at Fayoum University, El-Fayoum, Egypt.

${ }^{\mathrm{k}}$ Also at Ain Shams University, Cairo, Egypt.

${ }^{1}$ Also at British University in Egypt, Cairo, Egypt.

${ }^{\mathrm{m}}$ Also at Department of Physics, King Abdulaziz University, Jeddah, Saudi Arabia.

${ }^{\mathrm{n}}$ Also at Université de Haute Alsace, Mulhouse, France.

${ }^{\circ}$ Also at Skobeltsyn Institute of Nuclear Physics, Lomonosov Moscow State University, Moscow, Russia.

${ }^{\mathrm{p}}$ Also at Tbilisi State University, Tbilisi, Georgia.

${ }^{\mathrm{q}}$ Also at Ilia State University, Tbilisi, Georgia.

${ }^{r}$ Also at CERN, European Organization for Nuclear Research, Geneva, Switzerland.

${ }^{\mathrm{s}}$ Also at RWTH Aachen University, III. Physikalisches Institut A, Aachen, Germany.

${ }^{\mathrm{t}}$ Also at University of Hamburg, Hamburg, Germany.

uAlso at Brandenburg University of Technology, Cottbus, Germany.

${ }^{v}$ Also at MTA-ELTE Lendület CMS Particle and Nuclear Physics Group, Eötvös Loránd University, Budapest, Hungary.

${ }^{w}$ Also at Institute of Nuclear Research ATOMKI, Debrecen, Hungary.

${ }^{\mathrm{x}}$ Also at Institute of Physics, University of Debrecen, Debrecen, Hungary.

${ }^{\mathrm{y}}$ Also at IIT Bhubaneswar, Bhubaneswar, India.

${ }^{\mathrm{z}}$ Also at Institute of Physics, Bhubaneswar, India.

${ }^{\text {aa }}$ Also at Shoolini University, Solan, India.

${ }^{\mathrm{bb}}$ Also at University of Visva-Bharati, Santiniketan, India.

${ }^{\mathrm{cc}}$ Also at Isfahan University of Technology, Isfahan, Iran.

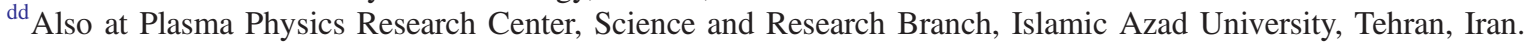

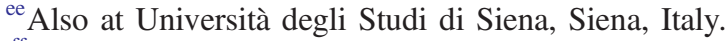

${ }^{\mathrm{ff}}$ Also at International Islamic University of Malaysia, Kuala Lumpur, Malaysia.

${ }^{\text {gg }}$ Also at Malaysian Nuclear Agency, MOSTI, Kajang, Malaysia.

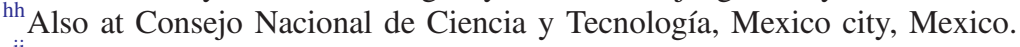

${ }^{\text {ii }}$ Also at Warsaw University of Technology, Institute of Electronic Systems, Warsaw, Poland.

${ }^{\mathrm{jj}}$ Also at Institute for Nuclear Research, Moscow, Russia.

${ }^{k k}$ Also at National Research Nuclear University 'Moscow Engineering Physics Institute' (MEPhI), Moscow, Russia.

${ }^{11}$ Also at St. Petersburg State Polytechnical University, St. Petersburg, Russia.

${ }^{\mathrm{mm}}$ Also at University of Florida, Gainesville, USA.

${ }^{n n}$ Also at P.N. Lebedev Physical Institute, Moscow, Russia.

${ }^{\text {oo }}$ Also at California Institute of Technology, Pasadena, USA.

${ }^{p p}$ Also at Budker Institute of Nuclear Physics, Novosibirsk, Russia.

${ }^{\mathrm{qq}}$ Also at Faculty of Physics, University of Belgrade, Belgrade, Serbia.

${ }^{\text {rr }}$ Also at INFN Sezione di Pavia, Università di Pavia, Pavia, Italy.

${ }^{\text {ss }}$ Also at University of Belgrade, Faculty of Physics and Vinca Institute of Nuclear Sciences, Belgrade, Serbia.

${ }^{\text {tt}}$ Also at Scuola Normale e Sezione dell'INFN, Pisa, Italy.

${ }^{\mathrm{uu}}$ Also at National and Kapodistrian University of Athens, Athens, Greece. 
${ }^{\mathrm{vv}}$ Also at Riga Technical University.

${ }^{w w}$ Also at Universität Zürich, Zurich, Switzerland.

${ }^{\mathrm{xx}}$ Also at Stefan Meyer Institute for Subatomic Physics.

${ }^{\mathrm{yy}}$ Also at Adiyaman University, Adiyaman, Turkey.

${ }^{\mathrm{zz}}$ Also at Istanbul Aydin University, Istanbul, Turkey.

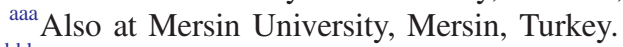

${ }^{b b b}$ Also at Piri Reis University, Istanbul, Turkey.

${ }^{c c c}$ Also at Gaziosmanpasa University, Tokat, Turkey.

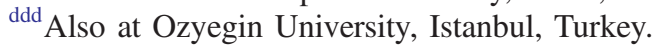

${ }^{e e e}$ Also at Izmir Institute of Technology, Izmir, Turkey.

${ }^{\mathrm{fff}}$ Also at Marmara University, Istanbul, Turkey.

${ }^{\text {ggg }}$ Also at Kafkas University, Kars, Turkey.

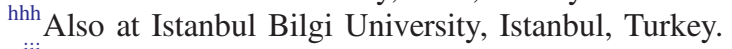

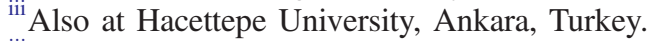

${ }_{\mathrm{jjj}}$ Also at Rutherford Appleton Laboratory, Didcot, United Kingdom.

${ }^{\mathrm{kkk}}$ Also at School of Physics and Astronomy, University of Southampton, Southampton, United Kingdom.

${ }^{111}$ Also at Monash University, Faculty of Science, Clayton, Australia.

${ }^{\mathrm{mmm}}$ Also at Bethel University.

${ }^{n n n}$ Also at Karamanoğlu Mehmetbey University, Karaman, Turkey.

${ }^{\circ 00}$ Also at Utah Valley University, Orem, USA.

${ }^{\mathrm{ppp}}$ Also at Purdue University, West Lafayette, USA.

${ }^{\mathrm{qqq}}$ Also at Beykent University.

${ }^{\mathrm{rrr}}$ Also at Bingol University, Bingol, Turkey.

${ }^{\text {sss }}$ Also at Sinop University, Sinop, Turkey.

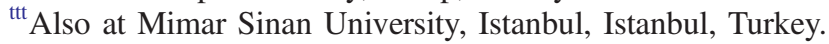

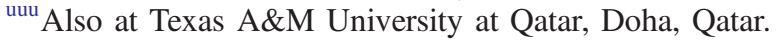

${ }^{\mathrm{vvv}}$ Also at Kyungpook National University. 